\title{
Synthesis of Cyclopentapeptides with Three to Five Aib Units
}

\author{
Arnhold, Franziska S ; Linden, Anthony ; Heimgartner, Heinz
}

\begin{abstract}
Four new Aib-containing cyclopentapeptides have been synthesized by cyclization of the corresponding linear pentapeptides using the diethyl phosphorocyanidate (DEPC)/EtN(iPr)2 method. The linear precursors were prepared via the 'azirine/oxazolone method', i.e., the Aib units were introduced by the reaction of amino acids or peptide acids with a 2,2-dimethyl-2H-azirin-3-amine, followed by selective hydrolysis of the terminal amide function. Most remarkably, cyclo[(Aib)5] exists in CDCl3 solution in a symmetrical conformation, i.e., no intramolecular H-bonds are detectable.
\end{abstract}

DOI: https://doi.org/10.1002/hlca.201400359

Posted at the Zurich Open Repository and Archive, University of Zurich ZORA URL: https://doi.org/10.5167/uzh-109170

Journal Article

Accepted Version

Originally published at:

Arnhold, Franziska S; Linden, Anthony; Heimgartner, Heinz (2015). Synthesis of Cyclopentapeptides with Three to Five Aib Units. Helvetica Chimica Acta, 98(2):232-244.

DOI: https://doi.org/10.1002/hlca.201400359 
15. 11.2014

Prof. Dr. H. Heimgartner

Tel. 0446354282

e-mail: heinz.heimgartner@chem.uzh.ch

\title{
Synthesis of Cyclopentapeptides with Three to Five Aib Units
}

\author{
by Franziska S. Arnhold ${ }^{1}$ ), Anthony Linden, and Heinz Heimgartner*
}

Institut für Chemie der Universität Zürich, Winterthurerstrasse 190, CH-8057 Zürich, Switzerland

${ }^{1}$ ) In part from the Ph.D. thesis of F. S. A., Universität Zürich, 1997. Present address: Bachem AG, Hauptstrasse 144, CH-4416 Bubendorf 
Four new Aib-containing cyclopentapeptides have been synthesized by cyclization of the corresponding linear pentapeptides using the DEPC/DIEA method. The linear precursors were prepared via the 'azirine/oxazolone method', i.e., the Aib units were introduced by the reaction of amino acids or peptide acids with a 2,2dimethyl-2H-azirin-3-amine, followed by selective hydrolysis of the terminal amide function. Most remarkably, cyclo(Aib) $)_{5}$ exists in $\mathrm{CDCl}_{3}$ solution in a symmetrical conformation, i.e., no intramolecular H-bonds are detectable. 
1. Introduction. - Although cyclopeptides have been known for many decades, their structures, syntheses and biological activities are of continuing interest (see refs. cited in [1]). With regards to their structures, cyclopeptides containing $\alpha$ aminoisobutyric acid (Aib) are of special concern because of the conformation determining properties of $\alpha, \alpha$-disubstituted $\alpha$-amino acids. Besides a few natural Aib-containing cyclopeptides [2], several examples have been synthesized, e.g., tetra[3], penta- [4], and hexapeptides [5], as well as examples with larger rings [6].

Our studies toward the use of 2,2-disubstituted $2 \mathrm{H}$-azirin-3-amines $\mathbf{1}$ as building blocks in the synthesis of peptides containing $\alpha, \alpha$-disubstituted glycines [7] proved that the 'azirine/oxazolone method' constitutes a convenient and efficient approach [8]. Based on this method, we have also prepared a series of cyclopeptides with Aib or other $\alpha, \alpha$-disubstituted glycines in their backbone [1][9]. As expected on the basis of structural studies of Aib-containing peptides [10], the $\beta$-turn motif is also a preferred structure of cyclopeptides with $\alpha, \alpha$-disubstituted glycines in the skeleton $[1][9][11]$.

In our recent publication [1], we described the cyclization of several pentapeptides with 2-methylphenylalanine (Phe(2Me)) and one or two Aib units, e.g. 2, to give the corresponding cyclopentapeptides, e.g., 3 (Scheme 1).

\section{Scheme 1}

In the present study, cyclopentapeptides with three to five Aib units were prepared. 
2. Results and Discussion. - The synthesis of the pentapeptides Z-Gly-AibAcb-Aib-Gly-OMe (4a, Acb = 1-aminocyclobutanecarboxylic acid) and Z-Gly-AibPro-Aib-Aib-N(Me)Ph (4b) have been described recently [12]. They were deprotected in the usual way: saponification of $4 \mathbf{a}$ with $\mathrm{LiOH} \cdot \mathrm{H}_{2} \mathrm{O}$ in $\mathrm{THF} / \mathrm{H}_{2} \mathrm{O} / \mathrm{MeOH}$ gave the peptide acid Z-Gly-Aib-Acb-Aib-Gly-OH (5a, 96\%), and subsequent hydrogenolysis, either with $\mathrm{H}_{2} / \mathrm{Pd} / \mathrm{C}$ in $\mathrm{MeOH}$ or with $\mathrm{HCO}_{2} \mathrm{NH}_{4} / \mathrm{Pd} / \mathrm{C}$ in $\mathrm{MeOH}$, led to H-Gly-Aib-Acb-Aib-Gly-OH (6a) in quantitative and 95\% yield, respectively. Selective hydrolysis of $\mathbf{4 b}$ with $3 \mathrm{~N} \mathrm{HCl}$ in $\mathrm{THF} / \mathrm{H}_{2} \mathrm{O}$ at room temperature yielded Z-Gly-Aib-Pro-Aib-Aib-OH (5b, 70\%) [12]. The latter was deprotected at the N-terminus to give H-Gly-Aib-Pro-Aib-Aib-OH (6b) quantitatively $\left(\mathrm{H}_{2} / \mathrm{Pd} / \mathrm{C}\right)$ and in $67 \%$ yield $\left(\mathrm{HCO}_{2} \mathrm{NH}_{4} / \mathrm{Pd} / \mathrm{C}\right)$, respectively.

The cyclization of the two pentapeptides occurred smoothly by treatment with DEPC)/DIEA) ${ }^{2}$ ) in DMF at room temperature to give the cyclopentapeptides $7 \mathbf{a}$ and 7b in 56 and $89 \%$ yield, respectively (Fig. 1). The structures were elucidated on the basis of the spectroscopic data in comparison with those of the previously reported analogues [1]. For example, the dominant peak in the ESI-MS of 7a appeared at $\mathrm{m} / \mathrm{z}$ $404\left([M+\mathrm{Na}]^{+}\right)$besides the $[M+1]^{+}$peak at $m / z$ 382. The ${ }^{1} \mathrm{H}-\mathrm{NMR}$ spectrum in $\mathrm{CDCl}_{3}$ showed four signals for $\mathrm{NH}$ groups at $7.21(s, 2 \mathrm{NH}), 7.08(t$-like, $1 \mathrm{NH}), 6.57$ and $6.23 \mathrm{ppm}(2 s, 2 \mathrm{NH})$ and only two $s$ for the Me groups of two Aib units. In the ${ }^{13} \mathrm{C}$ NMR spectrum $\left(\mathrm{CDCl}_{3}\right)$, five $\mathrm{C}=\mathrm{O}$ absorptions for lactam groups were present at 174.9, 174.8, 174.0, 171.8, and 171.4 ppm, and the Me groups of the two Aib units appeared at 25.2 and $24.7 \mathrm{ppm}$.

2) $\mathrm{DEPC}=$ diethyl phosphorocyanidate; DIEA = ethyl(diisopropyl)amine $\left(\mathrm{EtN}^{\mathrm{i}} \operatorname{Pr}_{2}\right.$, Hünig base). 
Fig. 1. Structures of the cyclopentapeptides $\mathbf{7 a}$ and $\mathbf{7 b}$.

For the attempted synthesis of cyclo(Gly-Aib-Aib-Aib-Aib) (7c), two linear pentapeptide precursors were prepared according to Schemes 2 and 3. Coupling of ZGly-OH with the known tetrapeptide amide 8 [13] using HBPyU/DIEA ${ }^{3}$ ) gave $\mathbf{4 c}$ in 98\% yield (Scheme 2). Deprotection of the latter in the usual way yielded 6c (90\%), which was cyclized by treatment with DEPC/DIEA at room temperature for $17 \mathrm{~h}$ to give $7 \mathrm{c}$ in $66 \%$ yield.

\section{Scheme 2}

In the first attempt to prepare the alternative precursor $\mathbf{6 d}$, the dipeptide acid $\mathbf{9}$ [13] was coupled with $\mathrm{H}-\mathrm{Gly}-\mathrm{OMe}$ to give, after saponification with $\mathrm{LiOH} \cdot \mathrm{H}_{2} \mathrm{O}$ in $\mathrm{MeOH}, \mathrm{Z}-(\mathrm{Aib})_{2}-\mathrm{Gly}-\mathrm{OH}(\mathbf{1 0})$ in $86 \%$ yield (Scheme 3). Subsequent coupling of the latter with $\left.\mathrm{H}-(\mathrm{Aib})_{2}-\mathrm{OMe}(\mathbf{1 1 a})^{4}\right)$, yielded the protected pentapeptide 4d $(74 \%)$, whose structure was established by X-ray crystallography (Fig. 2). Unexpectedly, all attempts to cleave the ester group by treatment with $\mathrm{LiOH} \cdot \mathrm{H}_{2} \mathrm{O}$ to give the pentapeptide acid failed. Therefore, the pentapeptide amide $\mathbf{4 e}$ was prepared by condensation of 10 with $\mathrm{H}-(\mathrm{Aib})_{2}-\mathrm{N}(\mathrm{Me}) \mathrm{Ph}(\mathbf{1 1 b})$, which was obtained from Z-

$\left.{ }^{3}\right) \mathrm{HBPyU}=O-($ Benzotriazol-1-yl $)-N, N, N^{\prime}, N^{\prime}$-bis(tetramethylen) uronium hexafluorophosphate

${ }^{4}$ ) Compound 11a was prepared from 9 via esterification with $\mathrm{MeOH} / \mathrm{BF}_{3} \cdot \mathrm{Et}_{2} \mathrm{O}$ followed by hydrogenolysis with $\mathrm{H}_{2} / \mathrm{Pd} / \mathrm{C}$ at room temperature in $86 \%$ yield. The product was contaminated with $c a$. $6 \%$ of 3,3,6,6-tetramethylpiperazine-2,5dione. 
(Aib) $)_{2}-\mathrm{N}(\mathrm{Me}) \mathrm{Ph}$ [13] by treatment with $\mathrm{H}_{2} / \mathrm{Pd} / \mathrm{C}$ in $\mathrm{MeOH}$. Selective cleavage of the terminal amide bond by treatment with $3 \mathrm{~N} \mathrm{HCl}$ in $\mathrm{THF} / \mathrm{H}_{2} \mathrm{O}$ at room temperature and subsequent deprotection of the N-terminus via transfer hydrogenolysis led to the desired pentapeptide $\mathbf{6 d}$ in $95 \%$ yield. The cyclization of $\mathbf{6 d}$ under the same conditions as used in the case of $\mathbf{6 c} \rightarrow \mathbf{7 c}$, but for $63 \mathrm{~h}$, gave the same cyclopentapeptide $\mathbf{7 c}$ but in the modest yield of $11 \%$.

\section{Scheme 3}

In the ESI-MS, characteristic peaks for cyclopentapeptide 7c were the $[M+1]^{+}$ peak at $m / z 398(110 \%)$ together with peaks for $[M+\mathrm{Na}]^{+}(\mathrm{m} / \mathrm{z}, 420)$ and $[M+\mathrm{K}]^{+}(\mathrm{m} / \mathrm{z}$ 436). The ${ }^{1} \mathrm{H}-\mathrm{NMR}$ spectrum in $\mathrm{CDCl}_{3}$ showed a signal for the lactam $\mathrm{NH}$ of Gly at $7.31 \mathrm{ppm}(t$-like) and four $s$ of Aib-NH at 7.05, 6.65, 6.29 and $6.25 \mathrm{ppm}$. The lactam $\mathrm{C}=\mathrm{O}$ groups appeared in the ${ }^{13} \mathrm{C}-\mathrm{NMR}$ spectrum ast $176.5,174.7,174.1,173.9$ and 172.4 ppm. The eight Me groups of the four Aib units absorbed as four $s$ in the ${ }^{1} \mathrm{H}$ NMR spectrum $(1.56,1.55,1.53,1.51 \mathrm{ppm})$ and three signals in the ${ }^{13} \mathrm{C}-\mathrm{NMR}$ spectrum $(25.5,24.9,24.8 \mathrm{ppm}(\mathrm{ca} .1: 2: 1))$. In addition, four $s$ for $\mathrm{C}(2)$ of four Aib and a $t$ for $\mathrm{CH}_{2}$ of Gly were detected in the ${ }^{13} \mathrm{C}$-NMR spectrum.

Fig. 2. ORTEP Plot [14] of the molecular structure of the pentapeptide 4d (50\% probability ellipsoids, arbitrary numbering of atoms, $\mathrm{H}$-atoms bonded to $\mathrm{C}$ atoms omitted for clarity)

Suitable crystals of $\mathbf{4 d}$ for an X-ray crystal-structure determination were obtained from AcOEt/hexane by slow evaporation of the solvent. The molecule 
adopts an overall helical conformation (Fig. 2). Each $\mathrm{NH}$ group of $\mathbf{4 d}$ acts as a donor for H-bonds (Table 1). Three of them are intramolecular ones, which form a regular pattern along the peptide chain: $\mathrm{N}(1)-\mathrm{H}, \mathrm{N}(4)-\mathrm{H}$ and $\mathrm{N}(7)-\mathrm{H}$ interact with the amide O-atom that is seven atoms further along the peptide backbone. Each of these interactions has a graph set motif [15] of $S(10)$, i.e., three $\beta$-turns of type III are formed (Table 2) leading to an overall $33_{10}$-helical conformation of the peptide. The remaining $\mathrm{N}(10)-\mathrm{H}$ and $\mathrm{N}(13)-\mathrm{H}$, which are not able to form such intramolecular $\mathrm{H}$ bonds because of their position in the backbone, form intermolecular H-bonds with the ester $\mathrm{C}=\mathrm{O}$ and amide $\mathrm{O}$-atoms, respectively, at the opposite end of the same neighboring molecule. These interactions link the molecules into extended chains running parallel to the $\left[10^{-}, 1\right]$ direction; graph sets $C(14)$ for each interaction.

Table 1. Intra- and intermolecular H-bonds of pentapeptide $4 \mathbf{d}$ (atom numbering refers to Fig. 2)

Table 2. Torsion angles $\phi, \psi$, and $\omega\left[^{\circ}\right]$ of the backbone of $\mathbf{4} \mathbf{d}$ in the crystal

Finally, the pentapeptide Z-(Aib) $)_{5} \mathrm{~N}(\mathrm{Me}) \mathrm{Ph}$ (4f) [13], prepared by repeated azirine coupling and selective hydrolysis, was deprotected to give $6 \mathbf{f}$ in $94 \%$ yield (Scheme 4). As $\mathbf{4 f}$ was rather insoluble in $\mathrm{THF} / \mathrm{H}_{2} \mathrm{O}$, the hydrolysis of the terminal amide group with $3 \mathrm{~N} \mathrm{HCl}$ had to be performed at $60^{\circ}$. The cyclization of $\mathbf{6} \mathbf{f}$ proved to be very difficult. Under the usual conditions, with DEPC/DIEA in DMF at room temperature as well as at $80^{\circ}$, mixtures of products were obtained, among them formylated pentapeptides, $\mathrm{H}-(\mathrm{Aib})_{5}-\mathrm{OMe}, \mathrm{H}-(\mathrm{Aib})_{10}-\mathrm{OMe}(\mathrm{MS})$, etc. Also in $\mathrm{CH}_{2} \mathrm{Cl}_{2}$ or in $\mathrm{MeCN}$ at reflux, only traces of the desired $\operatorname{cyclo}\left[(\mathrm{Aib})_{5}\right](\mathbf{7 d})$ could be detected 
(MS). Finally, after treatment with DEPC/DIEA in DMF at room temperature for 14 $\mathrm{d}$ and repeated chromatographic purification, $7 \mathbf{d}$ was obtained in $12 \%$ yield.

\section{Scheme 4}

The ESI-MS of 7d showed a dominant $[M+\mathrm{Na}]^{+}$peak $(100 \%)$ at $\mathrm{m} / \mathrm{z} 448$. Surprisingly, the ${ }^{1} \mathrm{H}-\mathrm{NMR}$ spectrum in $\mathrm{CDCl}_{3}$ showed only two $s$ at $6.63(\mathrm{NH})$ and $1.54 \mathrm{ppm}\left(\mathrm{Me}_{2} \mathrm{C}\right)$ with an intensity ratio of 1:6. Similarly, only three signals appeared

in the ${ }^{13} \mathrm{C}-\mathrm{NMR}$ spectrum $\left(\mathrm{CDCl}_{3}\right)$ : an $s$ at $175.2(\mathrm{C}=\mathrm{O}$ lactam $), s$ at $58.0(\mathrm{C}(2)$ of (Aib)), and $q$ at $25.0 \mathrm{ppm}\left(\mathrm{Me}_{2} \mathrm{C}\right.$ of Aib). From these data, we concluded that cyclo(Aib) $)_{5}(\mathbf{7 d})$ in $\mathrm{CDCl}_{3}$ solution exists in a perfectly symmetrical conformation, and no stable intramolecular H-bonds are formed.

3. Conclusions. - Three cyclopentapeptides containing three, four, and five Aib-units as well as cyclo(Gly-Aib-Acb-Aib-Gly) were prepared by cyclization of the corresponding linear precursors by treatment with DEPC/DIEA in DMF at room temperature. The syntheses of the linear pentapeptides were achieved using the 'azirine/oxazolone method' and segment condensation. The results of the cyclization reactions show once more (see introduction) that the efficiency of the ring closure strongly depends on steric factors, especially at the N-terminal amino acid, i.e., the nucleophile in the cyclization. Whereas cyclo[Gly-(Aib) $\left.)_{4}\right]$ was obtained in $66 \%$ yield from $\mathrm{H}-\mathrm{Gly}-(\mathrm{Aib})_{4}-\mathrm{OH}$, the yield dropped to $11 \%$ in the case of $\mathrm{H}-(\mathrm{Aib})_{2}-\mathrm{Gly}-(\mathrm{Aib})_{2}-$ $\mathrm{OH}$. Also, the ring closure of penta-Aib to give $c y c l o\left[(\mathrm{Aib})_{5}\right]$ occurred only sluggishly. 
Most remarkable are the NMR spectra of $\operatorname{cyclo}\left[(\mathrm{Aib})_{5}\right]$ as they indicate a symmetrical conformation, excluding the presence of stable intramolecular H-bonds.

Acknowledgement. - We thank the analytical sections of our institute for spectra and analyses, and the Stipendienfonds der Basler Chemischen Industrie and F. Hoffmann-La Roche AG, Basel, for financial support.

\section{Experimental part}

1. Abbreviations. Aib, 2-aminoisobutyric acid (2-methylalanin); DEPC, diethyl phosphorocyanidate; DIEA, ethyl(diisopropyl)amine (Hünig base); HBPyU, $O$-(benzotriazol-1-yl)- $N, N, N^{\prime}, N^{\prime}$-bis(tetramethylen)uronium hexafluorophosphate; Z, benzyloxycarbonyl.

2. General. See [1][12]. Solvents were purified by standard procedures. TLC: Merck TLC glass plates, silica gel $60 F_{254}$. Column chromatography $(\mathrm{CC})$ : UetikonChemie, silica gel C-560 (0.04-0.063 mm) or Merck 60, 0.040-0.063 mm. M.p.: Mettler-FP-5 apparatus, uncorrected. $[\alpha]_{\mathrm{D}}$-Values: Perkin-Elmer-241 polarimeter at $21^{\circ}$. IR Spectra: Perkin-Elmer-781 spectrometer, in $\mathrm{KBr} .{ }^{1} \mathrm{H}-$ and ${ }^{13} \mathrm{C}-\mathrm{NMR}$ spectra: Bruker AC-300, Bruker ARX-300 or Bruker AMX-600 spectrometer at 300 or 600 $\left({ }^{1} \mathrm{H}\right)$ and 75.5 or $150 \mathrm{MHz}\left({ }^{13} \mathrm{C}\right)$, respectively, in $\mathrm{CDCl}_{3}, \mathrm{CD}_{3} \mathrm{OD}$ or $\left(\mathrm{D}_{6}\right) \mathrm{DMSO}$; multiplicities of ${ }^{13} \mathrm{C}$ signals determined by the DEPT technique. ESI- and CI-MS: Finnigan TSQ-700 and Finnigan SSQ-700 instrument, respectively; $m / z$ (rel. \%).

General Procedure 1 (GP 1; Saponification of Peptide Methylesters). To a soln. of a peptide methyl ester $(1 \mathrm{mmol})$ in $10 \mathrm{ml}$ of $\mathrm{THF} / \mathrm{MeOH} / \mathrm{H}_{2} \mathrm{O}(3: 1: 1)$ at $0^{\circ}$ was added $\mathrm{LiOH} . \mathrm{H}_{2} \mathrm{O}(2.5 \mathrm{mmol})$. The mixture was stirred at $0^{\circ}$ for $1 \mathrm{~h}$. Then, it was 
neutralized by addition of aq. $2 \mathrm{~N} \mathrm{HCl}$ and the org. solvents were evaporated (rotavapor). The residue was dissolved in AcOEt und the mixture washed with aq. $0.5 \mathrm{~N} \mathrm{HCl}$. The org. phase was dried $\left(\mathrm{Na}_{2} \mathrm{SO}_{4}\right)$ and the solvent evaporated.

General Procedure 2 (GP 2; Hydrogenolysis). A mixture of Z-protected peptide in $\mathrm{MeOH}$ and $c a .10 \% \mathrm{Pd} / \mathrm{C}(10 \%)$ at r.t. was stirred under $\mathrm{H}_{2}$ (balloon) overnight. The mixture was filtered through a Celite pad and the solvent of the filtrate evaporated to dryness.

General Procedure 3 (GP 3; Transfer Hydrogenolysis). To a mixture of Zprotected peptide $(1 \mathrm{mmol})$ and the same amount of $\mathrm{Pd} / \mathrm{C}(10 \%)$ in $\mathrm{MeOH}$ was added $\mathrm{CO}_{2} \mathrm{NH}_{4}(5 \mathrm{mmol})$. The mixture was heated at reflux for $10 \mathrm{~min}$, the hot mixture filtered through a Celite pad and washed with $\mathrm{MeOH}$. The solvent of the filtrate was evaporated to dryness.

General Procedure 4 (GP 4; Hydrolysis of Peptide Amides). A soln. of Zprotected peptide amide $(1 \mathrm{mmol})$ in $3 \mathrm{~N} \mathrm{HCl}\left(\mathrm{THF} / \mathrm{H}_{2} \mathrm{O} 1: 1\right)$ was stirred at r.t. for 14.5 h. Then, $2 \mathrm{~N} \mathrm{HCl}$ was added and the mixture was extracted with $\mathrm{Et}_{2} \mathrm{O}$. The org. phase was dried $\left(\mathrm{Na}_{2} \mathrm{SO}_{4}\right)$, and the solvent was evaporated.

General Procedure 5 (GP 5; Cyclization with DEPC). To a ca. $1.5 \times 10^{-3} \mathrm{M}$ soln. of a deprotected pentapeptide $(0.1 \mathrm{mmol})$ in $\mathrm{DMF}(67 \mathrm{ml})$ at $0^{\circ}$ was added dropwise DEPC $(0.2-0.4 \mathrm{mmol})$ and DIEA $(1 \% \mathrm{v} / \mathrm{v})$, and the mixture was stirred overnight at r.t. Then, DMF was evaporated and the residue purified chromatographically and crystallized.

General Procedure 6 (GP 6, Segment Condensation). To a mixture of a Nprotected peptide (1 mmol), C-protected amino acid $(1.1 \mathrm{mmol})$, and HBPyU (1 mmol) in $\mathrm{CH}_{2} \mathrm{Cl}_{2}(1 \mathrm{ml})$ at r.t. was added DIEA (2 mmol; $3 \mathrm{mmol}$ in the case of an amino acid chloride) and the mixture stirred for $1 \mathrm{~h}$. Then, the solvent was 
evaporated, the residue was dissolved in AcOEt $(20 \mathrm{ml})$, washed with aq. $\mathrm{KHSO}_{4}$ $(5 \%, 3 \times)$, aq. $\mathrm{NaHCO}_{3}(5 \%, 3 \times)$, and aq. $\mathrm{NaCl}$ and purified by $\mathrm{CC}$.

3. Synthesis of cyclo(Gly-Aib-Acb-Aib-Gly) (7a). 3.1. Z-Gly-Aib-Acb-Aib-GlyOH (5a). Hydrolysis of Z-Gly-Aib-Acb-Aib-Gly-OMe (4a [12], 215 mg, 0.339 mmol) was performed with $\mathrm{LiOH} . \mathrm{H}_{2} \mathrm{O}(43 \mathrm{mg}, 1.025 \mathrm{mmol})$ in $\mathrm{THF} / \mathrm{H}_{2} \mathrm{O} / \mathrm{MeOH}(3: 1: 1,4$ ml) according to GP 1: $201 \mathrm{mg}(96 \%)$ of 5a. Colorless solid. M.p. 89.6-91.4 ${ }^{\circ}$ IR (KBr): 3300s (br), 3060m, 2990m, 2940m, 1755s, 1750s, 1740s, 1730s, 1715s, 1705s, $1695 s, 1670 s, 1660 s, 1650 s, 1645 s, 1555 s, 1540 s, 1535 s, 1525 s, 1505 m, 1470 m$, $1465 m, 1455 m, 1430 m, 1415 m, 1390 m, 1365 m, 1340 m, 1310 m, 1265 m, 1240 m$, 1185m, 1050m, 1015m, 740m, 695m. ${ }^{1} \mathrm{H}-\mathrm{NMR}\left(\left(\mathrm{D}_{6}\right) \mathrm{DMSO}\right): 8.41,8.10(2 s, 2 \mathrm{NH})$; 7.53 ( $t$-like, NH); 7.3-7.3 ( $m, 5$ arom. $\mathrm{H}, \mathrm{NH}) ; 7.25(s, \mathrm{NH}) ; 5.02\left(s, \mathrm{PhCH}_{2} \mathrm{O}\right) ; 3.7-$ $3.6\left(m, 2 \mathrm{CH}_{2}(\mathrm{Gly})\right) ; 2.55-2.4,2.1-2.0,1.9-1.75\left(3 m, 3 \mathrm{CH}_{2}\right.$ (Acb)); 1.36, $1.34(2 s, 2$ $\left.\mathrm{Me}_{2} \mathrm{C}\right) .{ }^{13} \mathrm{C}-\mathrm{NMR}\left(\left(\mathrm{D}_{6}\right) \mathrm{DMSO}\right):$ 174.7, 174.5, 172.5, 170.9, 169.8 (5s, $4 \mathrm{CO}$ (amide), $\mathrm{COOH}) ; 156.7$ (s, CO(urethane)); 136.8 ( $s, 1$ arom. C); 128.3, 127.7, $127.5(3 d, 5$ arom. $\mathrm{CH}) ; 65.5\left(t, \mathrm{PhCH}_{2} \mathrm{O}\right) ; 58.6,55.9,55.6$ (3s, $\left.2 \mathrm{C}(2)(\mathrm{Aib}), \mathrm{C}(2)(\mathrm{Acb})\right) ; 43.7$, $40.8\left(2 t, 2 \mathrm{CH}_{2}(\mathrm{Gly})\right) ; 30.4\left(t, 2 \mathrm{CH}_{2}(\mathrm{Acb})\right) ; 25.0,24.7\left(2 q, 2 \mathrm{Me}_{2} \mathrm{C}\right) ; 15.0(t$, $\left.\mathrm{CH}_{2}(\mathrm{Acb})\right)$. ESI-MS: $556\left(100,[M+\mathrm{Na}]^{+}\right), 459\left(3,[M-\mathrm{Gly}]^{+}\right), 374(10,[M-\mathrm{Aib}-$ Gly $\left.]^{+}\right)$.

3.2. H-Gly-Aib-Acb-Aib-Gly-OH (6a). a) Hydrogenolysis of 5a (233 mg, $0.437 \mathrm{mmol})$ in $\mathrm{MeOH}(10 \mathrm{ml})$ with $\mathrm{Pd} / \mathrm{C}(24 \mathrm{mg})$ according to $G P$ 2. After addition of $\mathrm{H}_{2} \mathrm{O}(10 \mathrm{ml})$ and filtration through Celite: $177 \mathrm{mg}$ (quant.) of $\mathbf{6 a}$.

b) Hydrogenolysis of $\mathbf{5 a}(114 \mathrm{mg}, 0.214 \mathrm{mmol})$ in $\mathrm{MeOH}$ according to $G P 3$ with $\mathrm{Pd} / \mathrm{C}(114 \mathrm{mg}): 81 \mathrm{mg}(95 \%)$ of 6a. Colorless solid. M.p. 239.2-240.7 $.{ }^{1} \mathrm{H}-$ $\operatorname{NMR}\left(\mathrm{D}_{2} \mathrm{O}\right): 3.83,3.75\left(2 s, 2 \mathrm{CH}_{2}(\mathrm{Gly})\right) ; 2.65-2.5,2.25-2.1,2.05-1.9(3 m, 3$ $\left.\mathrm{CH}_{2}(\mathrm{Acb})\right) ; 1.52,1.50\left(2 s, 2 \mathrm{Me}_{2} \mathrm{C}\right) .{ }^{13} \mathrm{C}-\mathrm{NMR}\left(\mathrm{D}_{2} \mathrm{O}\right): 179.7,179.0,177.9,169.4(4 s$, 
$4 \mathrm{CO}$ (amide), $\mathrm{COOH}) ; 62.1,59.5,59.4(3 s, 2 \mathrm{C}(2)(\mathrm{Aib}), \mathrm{C}(2)(\mathrm{Acb})) ; 46.3,43.3$ (2t, $\left.2 \mathrm{CH}_{2}(\mathrm{Gly})\right) ; 33.3\left(t, 2 \mathrm{CH}_{2}(\mathrm{Acb})\right) ; 27.1,26.7\left(2 q, 2 \mathrm{Me}_{2} \mathrm{C}\right) ; 17.7$ ( $\left.t, \mathrm{CH}_{2}(\mathrm{Acb})\right)$. ESIMS: $422\left(100,[M+N a]^{+}\right)$.

3.3. Cyclo(Gly-Aib-Acb-Aib-Gly) (7a). The cyclization of $6 \mathbf{a}(27.3 \mathrm{mg}, 0.068$ $\mathrm{mmol}$ ) in DMF (45 ml) was carried out with DEPC (125.5 mg, $0.77 \mathrm{mmol})$ and DIEA $(0.45 \mathrm{ml})$ according to $G P$ 5. After $\mathrm{CC}\left(\mathrm{CH}_{2} \mathrm{Cl}_{2} / \mathrm{MeOH} / \mathrm{NH}_{3}\right.$ 10:1:0.1) and crystallization from $\mathrm{CHCl}_{3} /$ hexane, $14.5 \mathrm{mg}(56 \%)$ of $7 \mathbf{a}$ were obtained. Colorless solid. M.p. 135.3-136.4 ${ }^{\circ}{ }^{1} \mathrm{H}-\mathrm{NMR}\left(\mathrm{CDCl}_{3}\right)$ : $7.21(s, 2 \mathrm{NH}) ; 7.08$ (t-like, $\left.\mathrm{NH}\right) ; 6.57$, $6.23(2 s, 2 \mathrm{NH}) ; 3.99\left(d, J=6.3, \mathrm{CH}_{2}(\mathrm{Gly})\right) ; 3.85\left(d, J=6.4, \mathrm{CH}_{2}(\mathrm{Gly})\right) ; 2.6-2.45$, 2.4-2.3, 2.15-2.0, 2.0-1.8 (4m, 2:2:1:1, $\left.3 \mathrm{CH}_{2}(\mathrm{Acb})\right)$; $1.58,1.55\left(2 s, 2 \mathrm{Me}_{2} \mathrm{C}\right) .{ }^{13} \mathrm{C}-$ NMR $\left(\mathrm{CDCl}_{3}\right)$ : 174.9, 174.8, 174.0, 171.8, 171.4 (5s, $5 \mathrm{CO}$ (amide)); 60.1, 57.7, 57.4 (3s, $2 \mathrm{C}(2)(\mathrm{Aib}), \mathrm{C}(2)(\mathrm{Acb})) ; 44.7,44.0\left(2 t, 2 \mathrm{CH}_{2}(\mathrm{Gly})\right) ; 31.1\left(t, 2 \mathrm{CH}_{2}(\mathrm{Acb})\right) ; 25.2$, $24.7\left(2 q, 2 \mathrm{Me}_{2} \mathrm{C}\right) ; 15.7\left(t, \mathrm{CH}_{2}(\mathrm{Acb})\right)$. ESI-MS: $404\left(100,[\mathrm{M}+\mathrm{Na}]^{+}\right), 382(7$, $\left.[M+1]^{+}\right)$.

4. Synthesis of cyclo(Gly-Aib-Pro-Aib-Aib) (7b). 4.1. H-Gly-Aib-Pro-Aib-Aib$\mathrm{OH}$ (6b). a) Hydrogenolysis of $\mathbf{5 b}$ [12] (86 mg, $0.153 \mathrm{mmol})$ in $\mathrm{MeOH}(3 \mathrm{ml})$ with $\mathrm{Pd} / \mathrm{C}(8.7 \mathrm{mg})$ according to $G P 2$. After $10 \mathrm{~min}$, the reaction was complete (TLC) and a precipitate formed, which dissolved again during stirring overnight: $66 \mathrm{mg}$ (quant.) of $6 \mathbf{b}$.

b) Hydrogenolysis of $\mathbf{5 b}(330 \mathrm{mg}, 0.588 \mathbf{m m o l})$ in $\mathrm{MeOH}$ (3 ml) according to GP 3 with $\mathrm{HCO}_{2} \mathrm{NH}_{4}(183 \mathrm{mg}, 2.902 \mathrm{mmol})$ and $\mathrm{Pd} / \mathrm{C}(331 \mathrm{mg}): 81 \mathrm{mg}(95 \%)$ of $\mathbf{6 b}$. Colorless solid. M.p. 173.6-175.3 . IR (KBr): 3320m, 3050m, 2980m, 2930m, $2870 m, 1670 s, 1620 s, 1550 s, 1470 m, 1450 m, 1415 m, 1390 m, 1360 m, 1280 m, 1210 m$, 1190m, 1170m. ${ }^{1} \mathrm{H}-\mathrm{NMR}\left(\mathrm{D}_{2} \mathrm{O}\right): 4.33$ (t-like, $\left.\mathrm{CH}(2)(\mathrm{Pro})\right) ; 3.89\left(s, \mathrm{CH}_{2}(\mathrm{Gly})\right) ; 3.8-$ 3.65, 3.6-3.5 (2m, $\left.\mathrm{CH}_{2}(5)(\mathrm{Pro})\right)$; 2.3-2.15, 2.1-1.8 (2m, 1:3, $\left.\mathrm{CH}_{2}(3), \mathrm{CH}_{2}(4)(\mathrm{Pro})\right)$; 
1.51, 1.48, 1.43, $1.42\left(4 s, 1: 3: 1: 1,3 \mathrm{Me}_{2} \mathrm{C}\right) .{ }^{13} \mathrm{C}-\mathrm{NMR}\left(\mathrm{D}_{2} \mathrm{O}\right): 184.5,178.2,176.7$, 176.4, 168.5 (5s, $4 \mathrm{CO}$ (amide), $\mathrm{COOH}) ; 65.7$ (d, $\mathrm{CH}(2)($ Pro $)$ ); 60.5, 59.6 (2s, 1:2, 3 $\mathrm{C}(2)(\mathrm{Aib})) ; 51.7\left(t, \mathrm{CH}_{2}(5)(\mathrm{Pro})\right) ; 42.8\left(t, \mathrm{CH}_{2}(\mathrm{Gly})\right) ; 31.0,28.3$ (2t, $\mathrm{CH}_{2}(3)$, $\mathrm{CH}_{2}$ (4)(Pro)); 27.2, 27.04, 26.97, 26.4 (4q, $\left.3 \mathrm{Me}_{2} \mathrm{C}\right)$. ESI-MS: $466\left(23,[\mathrm{M}+\mathrm{K}]^{+}, 450\right.$ $\left(100,[M+\mathrm{Na}]^{+}\right), 428\left(52,[M+1]^{+}\right), 325\left(16,[M-\mathrm{Aib}]^{+}\right), 286\left(20,[\text { Pro-Aib-Aib }]^{+}\right)$.

4.2. Cyclo(Gly-Aib-Pro-Aib-Aib) (7b). The cyclization of $\mathbf{6 b}(48.2 \mathrm{mg}, 0.113$ $\mathrm{mmol})$ in DMF (75 ml) was carried out with DEPC (67.0 mg, $0.411 \mathrm{mmol})$ and DIEA $(0.75 \mathrm{ml})$ according to $G P 5$. After $\mathrm{CC}\left(\mathrm{CH}_{2} \mathrm{Cl}_{2} / \mathrm{MeOH} 10: 1\right)$ and crystallization from AcOEt/hexane, $41.2 \mathrm{mg}(89 \%)$ of $\mathbf{7 b}$ were obtained. Colorless solid. M.p. 139.1$140.5^{\circ} \cdot[\alpha]_{\mathrm{D}}^{20}=-35.6(\mathrm{c}=0.95, \mathrm{EtOH}) . \mathrm{IR}(\mathrm{KBr}): 3300 \mathrm{~s}, 3040 \mathrm{~m}, 2980 \mathrm{~m}, 2940 \mathrm{~m}$, $1695 s, 1680 s, 1670 s, 1660 s, 1650 s, 1645 s, 1635 s, 1565 m, 1555 s, 1550 s, 1540 s$, $1520 s, 1505 s, 1480 m, 1470 m, 1460 m, 1455 m, 1445 m, 1390 s, 1365 s, 1270 m, 1245 m$, 1215m, 1195m, 1175m, 1075m, 1050m, 1030m. ${ }^{1} \mathrm{H}-\mathrm{NMR}\left(\left(\mathrm{D}_{6}\right) \mathrm{DMSO}\right): 8.7$ (very br. $s, \mathrm{NH}) ; 7.83$ (br. $s, \mathrm{NH}) ; 7.30$ (br. $s, \mathrm{NH}) ; 6.8$ (very br. $s, \mathrm{NH}) ; 4.5-4.35(m$, $\mathrm{CH}(2)(\mathrm{Pro})$ ); 4.15-3.8 ( $m, 1 \mathrm{H}$ of $\left.\mathrm{CH}_{2}(\mathrm{Gly})\right)$; $3.55-3.3$ ( $m, \mathrm{CH}_{2}$ (5)(Pro), $1 \mathrm{H}$ of $\mathrm{CH}_{2}$ (Gly)); 2.0-1.75 ( $\left.m, \mathrm{CH}_{2}(3), \mathrm{CH}_{2}(4)(\mathrm{Pro})\right) ; 1.49,1.44,1.40,1.31$ (4s, 1:2:2:1, 3 $\left.\mathrm{Me}_{2} \mathrm{C}\right)$. ESI-MS: $432\left(100,[M+\mathrm{Na}]^{+}\right), 410\left(20,[M+1]^{+}\right)$.

5. Synthesis of cyclo(Gly-Aib-Aib-Aib-Aib) (7c). 5.1. Via Cyclization of $H$ Gly-Aib-Aib-Aib-Aib-OH $\quad$ (6c). $\quad$ 5.1.1. Z-Gly-Aib-Aib-Aib-Aib-N(Me)Ph $\quad(\mathbf{4 c})$. According to GP 6, to a mixture of H-Aib-Aib-Aib-Aib-N(Me)Ph (8 [13], $202.6 \mathrm{mg}$, $0.453 \mathrm{mmol}$ ), Z-Gly-OH (98.2 mg, $0.469 \mathrm{mmol})$, and HBPyU (202.7 mg, 0.470 mmol) in $\mathrm{CH}_{2} \mathrm{Cl}_{2}(4 \mathrm{ml})$ at r.t. was added DIEA (119.7 mg, $\left.0.926 \mathrm{mmol}\right)$. After stirring for $21 \mathrm{~h}$, the precipitate was filtered and washed with a little $\mathrm{CH}_{2} \mathrm{Cl}_{2}$ to give 4c (243.7 mg) as a white powder. The filtrate was evaporated, and CC $\left(\mathrm{CH}_{2} \mathrm{Cl}_{2} / \mathrm{MeOH}\right)$ gave additional $40.7 \mathrm{mg}$ of 4c. Total yield: $284.4 \mathrm{mg}(98 \%)$. 
Colorless powder. M.p. 222.3-224.4․ IR (KBr): 3310m, 3280m, 2980m, 2940m, $1700 m, 1680 s, 1670 s, 1660 s, 1645 s, 1635 s, 1590 m, 1530 s, 1495 m, 1465 m, 1450 m$, 1395m, 1380m, 1360m, 1270m, 1230m, 1170m, 1090m, 710m. ${ }^{1} \mathrm{H}-\mathrm{NMR}\left(\mathrm{CD}_{3} \mathrm{OD}\right)$ : 7.4-7.2 (m, 10 arom. $\mathrm{H}) ; 5.10\left(s, \mathrm{PhCH}_{2} \mathrm{O}\right) ; 3.71\left(s, \mathrm{CH}_{2}(\mathrm{Gly})\right) ; 3.38(s, \mathrm{MeN}) ; 1.55$, 1.48, 1.41, $1.38\left(4 s, 4 \mathrm{Me}_{2} \mathrm{C}\right) .{ }^{13} \mathrm{C}-\mathrm{NMR}\left(\mathrm{CD}_{3} \mathrm{OD}\right): 177.1,176.7,176.4,175.9,172.1$ (5s, $5 \mathrm{CO}$ (amide)); 159.4 (s, $\mathrm{CO}$ (urethane)); 147.2, 138.2 (2s, 2 arom. C); 130.3, 129.6, 129.1, 128.8, 128.2, $128.1(6 d, 10$ arom. $\mathrm{CH}) ; 67.8\left(t, \mathrm{PhCH}_{2} \mathrm{O}\right) ; 58.4,58.2$, 57.9, $57.6(4 s, 4 \mathrm{C}(2)(\mathrm{Aib})) ; 45.2\left(t, \mathrm{CH}_{2}(\mathrm{Gly})\right) ; 41.1(q, \mathrm{MeN}) ; 26.3,26.0,25.34$, $25.27\left(4 q, 4 \mathrm{Me}_{2} \mathrm{C}\right)$. ESI-MS: $661\left(16,[M+\mathrm{Na}]^{+}\right), 532\left(100,[M-\mathrm{N}(\mathrm{Me}) \mathrm{Ph}]^{+}\right), 447$ $\left(49,[M-\mathrm{Aib}-\mathrm{N}(\mathrm{Me}) \mathrm{Ph}]^{+}\right), 362\left(18,[M-\mathrm{Aib}-\mathrm{Aib}-\mathrm{N}(\mathrm{Me}) \mathrm{Ph}]^{+}\right)$.

5.1.2. Z-Gly-Aib-Aib-Aib-Aib-OH (5c). According to GP 4, 4c $(255.8 \mathrm{mg}$, $0.400 \mathrm{mmol})$ was hydrolyzed in $3 \mathrm{~N} \mathrm{HCl}\left(\mathrm{THF} / \mathrm{H}_{2} \mathrm{O} 1: 1,10 \mathrm{ml}, 1 \mathrm{~h}\right)$. Extraction with AcOEt gave $213.4 \mathrm{mg}(97 \%)$ of 5c. A sample was recrystallized from boiling AcOEt. Colorless needles. M.p. 201.8-203.0. IR (KBr): 3340s, 3310s, 3300s, 3080m, 3070m, 3060m, 3040m, 2980m, 2940m, 2930m, 1730s, 1715s, 1710s, 1695s, 1680s, $1670 s, 1660 s, 1650 s, 1645 s, 1550 s, 1540 s, 1520 s, 1510 s, 1470 m, 1455 s, 1385 s$, $1365 m, 1280 s, 1240 s, 1230 s, 1170 s, 1160 s, 1155 s, 1050 m, 735 m, 700 m .{ }^{1} \mathrm{H}-\mathrm{NMR}$ $\left(\mathrm{CD}_{3} \mathrm{OD}\right)$ : 7.7-7.6 (m, 2 arom. $\left.\mathrm{H}\right) ; 7.4-7.3(m, 3$ arom. $\mathrm{H}) ; 5.10\left(s, \mathrm{PhCH}_{2} \mathrm{O}\right) ; 3.72(s$, $\mathrm{CH}_{2}$ (Gly)); 1.49, 1.42, 1.41, 1.39 (4s, $\left.4 \mathrm{Me}_{2} \mathrm{C}\right) .{ }^{13} \mathrm{C}-\mathrm{NMR}\left(\mathrm{CD}_{3} \mathrm{OD}\right): 178.3,176.9$, 176.6, 172.1 (4s, $4 \mathrm{CO}$ (amide), $\mathrm{COOH}) ; 159.8$ ( $s, \mathrm{CO}$ (urethane)); 138.2 (s, arom. C); 129.6, 129.1, $128.8(3 d, 5$ arom. $\mathrm{CH}) ; 67.9\left(t, \mathrm{PhCH}_{2} \mathrm{O}\right) ; 57.9,57.7,57.1(3 s, 4$ $\mathrm{C}(2)$ (Aib)); $45.3\left(t, \mathrm{CH}_{2}(\mathrm{Gly})\right) ; 25.7,25.41,25.36,25.3$ (4q, $\left.4 \mathrm{Me}_{2} \mathrm{C}\right)$. CI-MS: 551 (23), $550\left(79,[M+1]^{+}\right), 533(26), 532\left(93,[M-\mathrm{OH}]^{+}\right), 448(24), 447(100,[M-$ Aib$\left.\mathrm{OH}]^{+}\right), 442(32), 424(10), 416\left(18,\left[M-\mathrm{BnCO}_{2}\right]^{+}\right), 398(12), 362$ (32, [M-Aib-Aib- 
$\left.\mathrm{OH}]^{+}\right), 104\left(73,[\mathrm{H}-\mathrm{Aib}-\mathrm{OH}+1]^{+}\right)$. Anal. calc. for $\mathrm{C}_{24} \mathrm{H}_{36} \mathrm{~N}_{4} \mathrm{O}_{7} \cdot 0.5 \mathrm{H}_{2} \mathrm{O}$ (558.63): C 55.90, H 7.22, N 12.54; found: C 55.80, H 7.14, N 12.44.

5.1.3. H-Gly-Aib-Aib-Aib-Aib-OH (6c). According to $G P 3,5 \mathbf{c}(187.7 \mathrm{mg}$, $0.342 \mathrm{mmol})$ was deprotected by treatment with $\mathrm{HCO}_{2} \mathrm{NH}_{4}(109.8 \mathrm{mg}, 1.741 \mathrm{mmol})$ and $\mathrm{Pd} / \mathrm{C}(188.0 \mathrm{mg})$ in $\mathrm{MeOH}(7 \mathrm{ml}): 132.3 \mathrm{mg}(93 \%)$ of $\mathbf{6 c}$. Colorless solid. ${ }^{1} \mathrm{H}-$ $\operatorname{NMR}\left(\mathrm{D}_{2} \mathrm{O}\right): 3.84\left(s, \mathrm{CH}_{2}(\mathrm{Gly})\right) ; 1.48,1.47,1.43,1.42\left(4 s, 4 \mathrm{Me}_{2} \mathrm{C}\right) .{ }^{13} \mathrm{C}-\mathrm{NMR}$ $\left(\mathrm{D}_{2} \mathrm{O}\right): 179.0,178.8,178.5,169.3(4 s, 4 \mathrm{CO}($ amide $), \mathrm{COOH}) ; 60.6,59.7(2 s, 4$ $\mathrm{C}(2)(\mathrm{Aib})) ; 43.3\left(t, \mathrm{CH}_{2}(\mathrm{Gly})\right) ; 27.2,27.1,26.9,26.8$ (4q, $\left.4 \mathrm{Me}_{2} \mathrm{C}\right)$. CI-MS: 417 (29), $416\left(100,[M+1]^{+}\right), 398\left(14,[M-\mathrm{OH}]^{+}\right)$.

5.1.4. Cyclo(Gly-Aib-Aib-Aib-Aib) (7c). According to GP 5, to a soln. of $\mathbf{6 c}$ (38.6 mg, $0.093 \mathrm{mmol})$ in DMF (63 ml) were added DEPC (46.6 mg, $0.286 \mathrm{mmol})$ and DIEA $(0.6 \mathrm{ml})$ at r.t. After stirring for $17 \mathrm{~h}$, DMF was evaporated and the residue purified by $\mathrm{CC}\left(\mathrm{CH}_{2} \mathrm{Cl}_{2} / \mathrm{MeOH} 10: 1\right): 24.5 \mathrm{mg}$ (66\%) of 7c. Colorless solid. M.p. $126.3-127.7^{\circ} . \mathrm{IR}(\mathrm{KBr}): 3330 m, 2980 w, 2940 w, 1690 s, 1680 s, 1670 s, 1660 s, 1650 s$, $1645 s, 1635 s, 1550 s, 1540 s, 1530 s, 1515 s, 1505 s, 1470 m, 1460 m, 1390 m, 1375 m$, 1225m. ${ }^{1} \mathrm{H}-\mathrm{NMR}\left(\mathrm{CDCl}_{3}\right): 7.31(t$-like, $\mathrm{NH}) ; 7.05,6.65,6.29,6.25(4 s, 4 \mathrm{NH}) ; 3.39$ $\left(d, J=6.1, \mathrm{CH}_{2}(\mathrm{Gly})\right) ; 1.56,1.55,1.53,1.51\left(4 s, 4 \mathrm{Me}_{2} \mathrm{C}\right) .{ }^{13} \mathrm{C}-\mathrm{NMR}\left(\mathrm{CDCl}_{3}\right): 176.5$, 174.7, 174.1, 173.9, $172.4(5 s, 5 \mathrm{CO}$ (amide) $) ; 59.1,57.6,57.4,56.9(4 s, 4$ $\mathrm{C}(2)(\mathrm{Aib})) ; 45.1\left(t, \mathrm{CH}_{2}(\mathrm{Gly})\right) ; 25.5,24.9,24.8\left(3 q, 4 \mathrm{Me}_{2} \mathrm{C}\right)$. ESI-MS: 436 (12, $\left.[M+\mathrm{K}]^{+}\right), 420\left(19,[M+\mathrm{Na}]^{+}\right), 398\left(100,[M+1]^{+}\right)$.

5.2. Via Cyclization of H-Aib-Aib-Gly-Aib-Aib-OH (6d). 5.2.1. Z-Aib-AibGly-OMe. According to GP 6, with Z-Aib-Aib-OH (9 [13], $797.1 \mathrm{mg}, 2.473 \mathrm{mmol}$ ), H-Gly-OMe.HCl (343.1 mg, $2.733 \mathrm{mmol})$, HBPyU (1.070 g, $2.480 \mathrm{mmol})$ and DIEA (1.3 ml, $7.594 \mathrm{mmol})$ in $\mathrm{CH}_{2} \mathrm{Cl}_{2}(7 \mathrm{ml})$; reaction time $2.5 \mathrm{~h}$. The precipitate was filtered to give $550.3 \mathrm{mg}$ tripeptide; the filtrate was evaporated, and $\mathrm{CC}$ (AcOEt) 
gave an additional portion of tripeptide. Total yield: $906.8 \mathrm{mg}$ (93\%). Colorless solid. M.p. 151.5-152.2 ${ }^{\circ}$ IR (KBr): 3350s, 3280s, 3040m, 2980m, 2950m, 1755m, 1700s, $1670 s, 1660 s, 1535 s, 1520 s, 1455 m, 1435 m, 1410 m, 1385 m, 1370 m, 1265 m, 1215 s$, $1210 s, 1195 s, 1175 s, 1090 m, 1080 s, 985 m, 965 m, 755 m, 700 m .{ }^{1} \mathrm{H}-\mathrm{NMR}\left(\mathrm{CDCl}_{3}\right)$ : 7.42 (br. $s, \mathrm{NH}) ; 7.4-7.3(m, 5$ arom. $\mathrm{H}) ; 6.46,5.32(2 s, 2 \mathrm{NH}) ; 5.10\left(s, \mathrm{PhCH}_{2} \mathrm{O}\right)$; $3.99\left(d, J=5.6, \mathrm{CH}_{2}(\mathrm{Gly})\right) ; 3.72(s, \mathrm{MeO}) ; 1.48\left(s, 2 \mathrm{Me}_{2} \mathrm{C}\right) .{ }^{13} \mathrm{C}-\mathrm{NMR}\left(\mathrm{CDCl}_{3}\right)$ : 174.6, 173.1, 170.5 (3s, $2 \mathrm{CO}$ (amide), COOMe); 155.7 ( $s, \mathrm{CO}$ (urethane)); $136.0(s$, arom. C); 128.7, 128.5, $128.4(3 d, 5$ arom. $\mathrm{CH}) ; 67.1\left(t, \mathrm{PhCH}_{2} \mathrm{O}\right) ; 57.3,57.1(2 s, 2$ $\mathrm{C}(2)(\mathrm{Aib})) ; 51.9(q, \mathrm{MeO}) ; 41.4\left(t, \mathrm{CH}_{2}(\mathrm{Gly})\right) ; 25.43,25.38\left(2 q, 2 \mathrm{Me}_{2} \mathrm{C}\right) . \mathrm{CI}-\mathrm{MS}:$ 412 (6), $411\left(30,\left[M+\mathrm{NH}_{4}\right]^{+}\right), 395(21), 394\left(100,[M+1]^{+}\right), 331(14), 306(22), 305$ (8). Anal. calc. for $\mathrm{C}_{19} \mathrm{H}_{27} \mathrm{~N}_{3} \mathrm{O}_{6}$ (393.44): C 58.00, H 6.92, N 10.68; found: C 57.88, H 7.04, N 10.62.

5.2.2. Z-Aib-Aib-Gly-OH (10). According to GP 1, Z-Aib-Aib-Gly-OMe (906.8 mg, $2.305 \mathrm{mmol}$ ) was saponified with $\mathrm{LiOH} . \mathrm{H}_{2} \mathrm{O}(243.0 \mathrm{mg}, 5.791 \mathrm{mmol})$ in THF/MeOH/ $\mathrm{H}_{2} \mathrm{O}$ (3:1:1, $\left.7 \mathrm{ml}\right): 809 \mathrm{mg}$ (93\%). Colorless solid. IR (KBr): 3320s, 3300s, 3085m, 3030m, 3000m, 2940m, 2910m, 2900m, 1765m, 1750m, 1702s, 1665s, $1620 s, 1615 s, 1565 s, 1515 s, 1470 m, 1450 m, 1410 m, 1385 m, 1370 m, 1335 m, 1265 s$, 1220m, 1175s, 1085s, 900m, 740m, 695m. ${ }^{1} \mathrm{H}-\mathrm{NMR}\left(\mathrm{CD}_{3} \mathrm{OD}\right): 7.90$ (br. $\left.s, \mathrm{NH}\right) ; 7.74$ $(s, \mathrm{NH}) ; 7.4-7.25(m, 5$ arom. $\mathrm{H}) ; 5.09\left(s, \mathrm{PhCH}_{2} \mathrm{O}\right) ; 3.89-3.87\left(m, \mathrm{CH}_{2}(\mathrm{Gly})\right) ; 1.42$, $1.39\left(2 s, 2 \mathrm{Me}_{2} \mathrm{C}\right) .{ }^{13} \mathrm{C}-\mathrm{NMR}\left(\mathrm{CD}_{3} \mathrm{OD}\right): 177.6,176.7,173.0$ (3s, $2 \mathrm{CO}$ (amide), $\mathrm{COOH}) ; 158.1$ (s, CO(urethane)); 138.2 ( $s$, arom. C); 129.6, 129.24, $129.18(3 d, 5$ arom. $\mathrm{CH}) ; 67.8\left(t, \mathrm{PhCH}_{2} \mathrm{O}\right) ; 58.0,57.8(2 s, 2 \mathrm{C}(2)(\mathrm{Aib})) ; 42.0\left(t, \mathrm{CH}_{2}(\mathrm{Gly})\right) ; 25.4$ $\left(q, 2 \mathrm{Me}_{2} \mathrm{C}\right) . \mathrm{CI}-\mathrm{MS}: 398(6), 397\left(36,\left[M+\mathrm{NH}_{4}\right]^{+}\right), 381(19), 380\left(100,[M+1]^{+}\right), 305$ (15, $\left.[M-\mathrm{Gly}]^{+}\right), 289(17), 272(16), 246\left(30,[M-\mathrm{BnOCO}]^{+}\right.$. 
5.2.3. Z-Aib-Aib-OMe [16]. To a soln. of Z-Aib-Aib-OH [13] (1.070 g, 3.317 mmol $)$ in $\mathrm{MeOH}(30 \mathrm{ml})$ was added $\mathrm{BF}_{3} \cdot \mathrm{Et}_{2} \mathrm{O}(0.42 \mathrm{ml})$ and the mixture stirred for 18 h. The solvent was evaporated, the residue dissolved in $\mathrm{CH}_{2} \mathrm{Cl}_{2}(150 \mathrm{ml})$, and the mixture extracted with aq. $2 \mathrm{~N} \mathrm{HCl}(2 \times)$, aq. $1 \mathrm{~N} \mathrm{NaOH}(2 \times)$ and brine, dried $\left(\mathrm{MgSO}_{4}\right)$, and the solvent evaporated. Crystallization from AcOEt/hexane yielded Z-Aib-AibOMe (1.075 g, 97\%). Colorless solid. M.p. 107.4-108.6 . IR (KBr): 3380m, 3360m, $3320 s, 3280 s, 3030 m, 2980 m, 2950 m, 1730 s, 1715 s, 1660 s, 1545 m, 1535 s, 1520 s$, $1470 m, 1465 m, 1450 m, 1435 m, 1385 m, 1360 m, 1300 m, 1255 s, 1225 m, 1215 m$, $1190 m, 1170 m, 1155 m, 1085 m, 1070 s, 960 m, 745 m, 695 m .{ }^{1} \mathrm{H}-\mathrm{NMR}\left(\mathrm{CDCl}_{3}\right): 7.4-$ $7.3(m, 5$ arom. H); 6.90 (br. $s, 1 \mathrm{NH}) ; 5.31$ (br. $s, 1 \mathrm{NH}) ; 5.09\left(s, \mathrm{PhCH}_{2} \mathrm{O}\right) ; 3.72(s$, $\mathrm{MeO}) ; 1.51,1.50\left(2 s, 2 \mathrm{Me}_{2} \mathrm{C}\right) .{ }^{13} \mathrm{C}-\mathrm{NMR}\left(\mathrm{CDCl}_{3}\right): 175.0,173.5$ (2s, $\mathrm{CO}$ (amide), COOMe); 155.1 ( $s, \mathrm{CO}($ urethane)); 136.3 ( $s$, arom. C); 128.5, 128.2, 128.1 (3d, 5 arom. $\mathrm{CH}) ; 66.7\left(t, \mathrm{PhCH}_{2} \mathrm{O}\right) ; 57.0,56.5$ (2s, $\left.2 \mathrm{C}(2)(\mathrm{Aib})\right) ; 52.2(q, \mathrm{MeO}) ; 25.4,24.5$ (2q, $\left.2 \mathrm{Me}_{2} \mathrm{C}\right)$. CI-MS: 338 (17), $337\left(100,[M+1]^{+}\right), 230$ (5), 229 (59). Anal. calc. for $\mathrm{C}_{17} \mathrm{H}_{24} \mathrm{~N}_{2} \mathrm{O}_{5}$ (336.39): C 60.70, H 7.19, N 8.33; found: C 60.92, H 7.38, N 8.36.

5.2.4. H-Aib-Aib-OMe [16] (11a). According to GP2, Z-Aib-Aib-OMe (204.8 $\mathrm{mg}, 0.609 \mathrm{mmol}) \mathrm{im} \mathrm{MeOH}(3 \mathrm{ml})$ was deprotected (19.6 mg Pd/C, $30 \mathrm{~min}): 109.5$ mg (89\%) of 11a (contaminated with ca. 6\% of 3,3,6,6-tetramethylpiperazine-2,5dione [17]). ${ }^{1} \mathrm{H}-\mathrm{NMR}\left(\left(\mathrm{D}_{6}\right) \mathrm{DMSO}\right): 8.03$ ( $\left.s, \mathrm{NH}\right) ; 3.57$ (s, MeO); 2.45-1.7 (br. signal, $\left.\mathrm{NH}_{2}\right) ; 1.38,1.15\left(2 s, 2 \mathrm{Me}_{2} \mathrm{C}\right) .{ }^{13} \mathrm{C}-\mathrm{NMR}\left(\left(\mathrm{D}_{6}\right) \mathrm{DMSO}\right): 177.0,174.4(2 s$, CO(amide), COOMe); 54.7, 54.0 (2s, 2 C(2)(Aib)); 51.7 ( $q$, MeO); 28.3, 24.6 (2q, 2 $\left.M e_{2} \mathrm{C}\right)$. CI-MS: $405\left(23,[2 M+1]^{+}\right), 204(9), 203\left(100,[M+1]^{+}\right)$.

5.2.5. $H$-Aib-Aib-N(Me)Ph (11b). According to GP2, Z-Aib-Aib-N(Me)Ph [13] (613 mg, $1.490 \mathrm{mmol}) \mathrm{im} \mathrm{MeOH} \mathrm{(2} \mathrm{ml)} \mathrm{was} \mathrm{deprotected} \mathrm{(10} \mathrm{mg} \mathrm{Pd/C,} 30 \mathrm{~min})$ : $413 \mathrm{mg}$ (quant.) of 11b. Colorless oil. ${ }^{1} \mathrm{H}-\mathrm{NMR}\left(\mathrm{CDCl}_{3}\right)$ : 7.68 (br. $\left.s, \mathrm{NH}\right) ; 7.45-7.25$ 
(m, 5 arom. H); 3.27 (s, MeN); 1.48 (br. $\left.s, \mathrm{NH}_{2}\right) ; 1.49,1.25$ (2s, $\left.2 \mathrm{Me}_{2} \mathrm{C}\right) .{ }^{13} \mathrm{C}-\mathrm{NMR}$ $\left(\mathrm{CDCl}_{3}\right):$ 175.9, $173.4(2 s, 2 \mathrm{CO}($ amide $)) ; 145.2$ ( $s$, arom. C); 129.3, 128.0, $127.6(3 d$, 5 arom. $\mathrm{CH}) ; 57.4,54.9(2 s, 2 \mathrm{C}(2)(\mathrm{Aib})) ; 41.4(q, \mathrm{MeN}) ; 28.8,26.6\left(2 q, 2 \mathrm{Me}_{2} \mathrm{C}\right)$.

5.2.6. Z-Aib-Aib-Gly-Aib-Aib-OMe (4d). According to GP 6, with $\mathbf{1 0}$ (138.8 mg, $0.366 \mathrm{mmol})$, freshly prepared 11a $(82.3 \mathrm{mg}, 0.407 \mathrm{mmol})$, HBPyU (158.2 mg, $0.367 \mathrm{mmol})$, and DIEA (131 $\mathrm{mg}, 1.014 \mathrm{mmol})$ in $\mathrm{CH}_{2} \mathrm{Cl}_{2}(3 \mathrm{ml})$; reaction time $2 \mathrm{~h}$. The precipitate formed during evaporation was filtered to give $103.2 \mathrm{mg} \mathbf{4 d}$; the filtrate was evaporated, and CC (AcOEt) gave an additional portion of 4d. Total yield: $151.9 \mathrm{mg}$ (74\%). Colorless solid. M.p. 175.3-176.1 ${ }^{\circ}$. IR (KBr): 3350s, 3320s, $3300 s, 3260 s, 3040 m, 2980 m, 2940 m, 1760 s, 1700 s, 1680 s, 1665 s, 1660 s, 1650 s$, $1540 s, 1515 s, 1470 m, 1465 m, 1455 m, 1410 m, 1385 m, 1360 m, 1305 m, 1265 s, 1230 m$, 1215m, 1195m, 1160m, 1080s, $700 m .{ }^{1} \mathrm{H}-\mathrm{NMR}\left(\mathrm{CDCl}_{3}\right): 7.74$ (t-like, NH); 7.33 (br. $s, 5$ arom. $\mathrm{H}, \mathrm{NH}) ; 7.08,6.55,6.05(3 s, 3 \mathrm{NH}) ; 5.09\left(s, \mathrm{PhCH}_{2} \mathrm{O}\right) ; 3.70(d, J=5.8$, $\left.\mathrm{CH}_{2}(\mathrm{Gly})\right) ; 3.66(s, \mathrm{MeO}) ; 1.53,1.51,1.48,1.39$ (4s, $\left.4 \mathrm{Me}_{2} \mathrm{C}\right) .{ }^{13} \mathrm{C}-\mathrm{NMR}\left(\mathrm{CDCl}_{3}\right)$ : 175.5, $175.3, \quad 174.5, \quad 174.3, \quad 169.0 \quad(5 s, 4$ CO(amide), COOMe); $156.5 \quad(s$, $\mathrm{CO}($ urethane)); 136.0 (s, arom. C); 128.7, 128.6, 128.1 (3d, 5 arom. $\mathrm{CH}) ; 67.5(t$, $\left.\mathrm{PhCH}_{2} \mathrm{O}\right) ; 57.1,56.7,55.8$ (3s, $\left.4 \mathrm{C}(2)(\mathrm{Aib})\right) ; 52.1$ ( $\left.q, \mathrm{MeO}\right) ; 44.7\left(t, \mathrm{CH}_{2}(\mathrm{Gly})\right) ; 25.4$, 25.13, 25.08, $25.0\left(4 q, 4 \mathrm{Me}_{2} \mathrm{C}\right)$. ESI-MS: $586\left(100,[M+\mathrm{Na}]^{+}\right)$. Anal. calc. for $\mathrm{C}_{27} \mathrm{H}_{41} \mathrm{~N}_{5} \mathrm{O}_{8}$ (563.65): C 57.54, H 7.43, N 12.42; found: C 57.54, H 7.20, N 12.21.

Crystals suitable for an X-ray crystal-structure determination were grown from AcOEt/hexane by slow evaporation of the solvent.

5.2.7. Z-Aib-Aib-Gly-Aib-Aib-N(Me)Ph (4e). According to GP 6, with $\mathbf{1 0}$ (386.5 mg, $1.019 \mathrm{mmol}$ ), 11b (338.2 mg, $1.219 \mathrm{mmol}$ ), HBPyU (442 mg, 1.025 mmol), and DIEA (309 mg, $2.391 \mathrm{mmol})$ in $\mathrm{CH}_{2} \mathrm{Cl}_{2}(5 \mathrm{ml})$; reaction time $16 \mathrm{~h}$. Purification by $\mathrm{CC}\left(\mathrm{CH}_{2} \mathrm{Cl}_{2} / \mathrm{MeOH} 20: 1\right)$ gave $529.8 \mathrm{mg}(81 \%)$ of 4e. A sample was 
recrystallized from $\mathrm{CH}_{2} \mathrm{Cl}_{2}$ /hexane. Colorless crystals. M.p. 199.7-201.4 ${ }^{\circ}$. IR (KBr): $3310 s, 3250 m, 2980 m, 1690 s, 1680 s, 1660 s, 1645 s, 1595 m, 1495 m, 1470 m, 1455 m$, $1435 m, 1395 m, 1385 m, 1360 m, 1270 s, 1215 m, 1195 m, 1175 m, 1090 m, 1070 m, 700 m$. ${ }^{1} \mathrm{H}-\mathrm{NMR}\left(\mathrm{CD}_{3} \mathrm{OD}\right): 7.45-7.25$ ( $m, 10$ arom. $\left.\mathrm{H}\right) ; 5.11\left(s, \mathrm{PhCH}_{2} \mathrm{O}\right) ; 3.62(s$, $\left.\mathrm{CH}_{2}(\mathrm{Gly})\right) ; 3.36$ (s, MeN); 1.53, 1.51, 1.41, 1.35 (4s, $\left.4 \mathrm{Me}_{2} \mathrm{C}\right) .{ }^{13} \mathrm{C}-\mathrm{NMR}\left(\mathrm{CD}_{3} \mathrm{OD}\right)$ : 178.5, 177.4, 176.5, 175.6, 171.8 (5s, $5 \mathrm{CO}$ (amide)); 158.5 ( $s, \mathrm{CO}$ (urethane)); 147.0, 138.5 (2s, 2 arom. C); 130.3, 129.6, 129.2, 128.9, 128.4 (5d, 10 arom. $\mathrm{CH}) ; 68.0(t$, $\left.\mathrm{PhCH}_{2} \mathrm{O}\right) ;$ 58.5, $57.2(2 s, 4 \mathrm{C}(2)(\mathrm{Aib})) ; 45.3\left(t, \mathrm{CH}_{2}(\mathrm{Gly})\right) ; 41.1(q, \mathrm{MeN}) ; 26.4,26.0$, 25.4, $25.3\left(4 q, 4 M e_{2} \mathrm{C}\right)$. ESI-MS: $661\left(100,[M+\mathrm{Na}]^{+}\right), 532\left(6,[M-\mathrm{N}(\mathrm{Me}) \mathrm{Ph}]^{+}\right)$.

5.2.8. Z-Aib-Aib-Gly-Aib-Aib-OH. According to GP 4, 4e (448.5 mg, 0.702 mmol) was hydrolyzed in $3 \mathrm{~N} \mathrm{HCl}\left(\mathrm{THF} / \mathrm{H}_{2} \mathrm{O} 1: 1 ; 7 \mathrm{ml}\right): 370.2 \mathrm{mg}(96 \%)$ of Z-AibAib-Gly-Aib-Aib-OH. Colorless solid. M.p. 215.4-216.8 . IR (KBr): 3300s, 3060m, $3040 m, 2990 m, 2940 m, 1720 s, 1695 s, 1670 s, 1660 s, 1550 m, 1540 s, 1530 s, 1525 s$, $1470 m, 1460 m, 1455 m, 1385 m, 1365 m, 1270 s, 1220 m, 1170 m, 1090 m, 1080 m, 700 m$. ${ }^{1} \mathrm{H}-\mathrm{NMR}\left(\mathrm{CD}_{3} \mathrm{OD}\right)$ : 7.45-7.3 (m, 5 arom. H); $5.11\left(s, \mathrm{PhCH}_{2} \mathrm{O}\right) ; 3.64\left(s, \mathrm{CH}_{2}(\mathrm{Gly})\right)$; 1.49, 1.48, 1.41, $1.36\left(4 s, 4 \mathrm{Me}_{2} \mathrm{C}\right) .{ }^{13} \mathrm{C}-\mathrm{NMR}\left(\mathrm{CD}_{3} \mathrm{OD}\right): 178.3,178.0,177.4,176.2$, $171.8(5 s, 4 \mathrm{CO}$ (amide), $\mathrm{COOH}) ; 158.5$ ( $s, \mathrm{CO}$ (urethane)); 138.5 ( $s$, arom. C); 129.6, 129.2, $128.9(3 d, 5$ arom. $\mathrm{CH}) ; 68.0\left(t, \mathrm{PhCH}_{2} \mathrm{O}\right)$; 58.2, 57.8, 57.7, $57.1(4 s, 4$ $\mathrm{C}(2)(\mathrm{Aib})) ; 45.0\left(t, \mathrm{CH}_{2}(\mathrm{Gly})\right) ; 25.7,25.4,25.30,25.27$ (4q, $\left.4 \mathrm{Me}_{2} \mathrm{C}\right)$. ESI-MS: 572 $\left(100,[M+\mathrm{Na}]^{+}\right)$

5.2.9. H-Aib-Aib-Gly-Aib-Aib-OH (6d). According to GP 3, Z-Aib-Aib-GlyAib-Aib-OH (281.5 mg, $0.513 \mathrm{mmol})$ was deprotected in $\mathrm{MeOH}(9 \mathrm{ml})$ by treatment with $\mathrm{HCO}_{2} \mathrm{NH}_{4}(163 \mathrm{mg}, 2.585 \mathrm{mmol})$ and $\mathrm{Pd} / \mathrm{C}(280.3 \mathrm{mg}): 214.0 \mathrm{mg}$ (quant.) of 6d. Colorless solid. ${ }^{1} \mathrm{H}-\mathrm{NMR}\left(\mathrm{D}_{2} \mathrm{O}\right): 3.85\left(s, \mathrm{CH}_{2}(\mathrm{Gly})\right)$; 1.64, 1.51, 1.49, 1.43 (4s, 4 $\left.\mathrm{Me}_{2} \mathrm{C}\right) .{ }^{13} \mathrm{C}-\mathrm{NMR}\left(\mathrm{CD}_{3} \mathrm{OD}\right): 184.7,179.9,177.8,175.2,173.2$ (5s, $4 \mathrm{CO}$ (amide), 
$\mathrm{COOH}) ; 60.8,60.0,59.3(3 s, 4 \mathrm{C}(2)(\mathrm{Aib})) ; 46.0\left(t, \mathrm{CH}_{2}(\mathrm{Gly})\right) ; 27.1,26.9,26.7,25.9$ $\left(4 q, 4 \mathrm{Me}_{2} \mathrm{C}\right) . \mathrm{ESI}-\mathrm{MS}: 416\left(100,[M+1]^{+}\right)$.

5.2.10. Cyclo(Gly-Aib-Aib-Aib-Aib) (7c). According to GP 5, to a soln. of $\mathbf{6 d}$ (27.2 mg, $0.066 \mathrm{mmol})$ in DMF (45 ml) were added DEPC (34.0 mg, $0.21 \mathrm{mmol})$ and DIEA $(0.4 \mathrm{ml})$ at r.t. After stirring for $63 \mathrm{~h}$, DMF was evaporated and the residue purified by $\mathrm{CC}\left(\mathrm{CH}_{2} \mathrm{Cl}_{2} / \mathrm{MeOH} 20: 1 \rightarrow 10: 1\right): 2.8 \mathrm{mg}(11 \%)$ of $7 \mathbf{c}$.

6. Synthesis of cyclo(Aib-Aib-Aib-Aib-Aib) (7d). 6.1. Z-Aib-Aib-Aib-Aib-Aib$O H$. According to GP 4, Z-Aib-Aib-Aib-Aib-Aib-N(Me)Ph (4f [13], $881.1 \mathrm{mg}, 1.321$ mmol) was hydrolyzed at $60^{\circ}$ for $1 \mathrm{~h}$. The precipitate was filtered and washed with $\mathrm{Et}_{2} \mathrm{O}$ to give $726.8 \mathrm{mg}$ of Z-Aib-Aib-Aib-Aib-Aib-OH. Extraction of the aq. phase with $\mathrm{Et}_{2} \mathrm{O}(4 \times)$ gave additional $34.0 \mathrm{mg}$ of the product. Total yield: $760.8 \mathrm{mg}$ (quant.). Colorless solid. M.p. 226.2-227.3․ IR (KBr): 3410s, 3320s, 3290s, 3250s, 3040s, 2980s, 2940s, 2920m, 2900m, 1745s, 1730s, 1710s, 1705s, 1695s, 1680s, $1675 s, 1665 s, 1645 s, 1635 s, 1580 m, 1565 m, 1550 s, 1530 s, 1515 s, 1465 s, 1455 s$, $1445 s, 1385 s, 1365 s, 1320 s, 1315 s, 1280 s, 1270 s, 1265 s, 1225 s, 1210 s, 1185 s$, 1180s, 1170s, 1165s, 1090s, 1080s, 955m, 945m, 750m, 700m. ${ }^{1} \mathrm{H}-\mathrm{NMR}$ ((D)DMSO): 11.83 (br. $s, \mathrm{COOH}) ; 8.23,7.85,7.46$ (3s, $3 \mathrm{NH})$; 7.4-7.3 (m, 5 arom. $\mathrm{H}, 1 \mathrm{NH}) ; 7.28(s, \mathrm{NH}) ; 5.09\left(s, \mathrm{PhCH}_{2} \mathrm{O}\right) ; 1.33,1.32,1.21\left(3 s, 3: 1: 1,5 \mathrm{Me}_{2} \mathrm{C}\right) .{ }^{13} \mathrm{C}-$ NMR ((D 6$)$ DMSO): 175.5, 174.9, 174.7, 173.42, 173.38 (5s, 4 CO(amide), $\mathrm{COOH})$; 155.7 ( $s, \mathrm{CO}$ (urethane)); 137.1 ( $s$, arom. C); 128.3, 127.6, 127.1 (3d, 5 arom. $\mathrm{CH})$; $65.4\left(t, \mathrm{PhCH}_{2} \mathrm{O}\right) ; 55.9,55.81,55.76,55.6,54.5$ (5s, $\left.5 \mathrm{C}(2)(\mathrm{Aib})\right) ; 25.0,24.6,24.5$, $24.3\left(4 q, 5 \mathrm{Me}_{2} \mathrm{C}\right)$. ESI-MS: $660\left(100,[\mathrm{M+Na}]^{+}\right), 578\left(80,[M+1]^{+}\right), 560(8,[M-$ $\left.\mathrm{OH}]^{+}\right), 475\left(19,[M-\mathrm{Aib}-\mathrm{OH}]^{+}\right)$. Anal. calc. for $\mathrm{C}_{28} \mathrm{H}_{43} \mathrm{~N}_{5} \mathrm{O}_{8}$ (577.68): C 58.22, H 7.50, N 12.12; found: C 58.36, H 7.30, N 11.93. 
6.2. $H$-Aib-Aib-Aib-Aib-Aib-OH (6f). According to GP 3, Z-Aib-Aib-AibAib-Aib-OH (233.2 mg, $0.404 \mathrm{mmol})$ in $\mathrm{MeOH}(7 \mathrm{ml})$ was deprotected by treatment with $\mathrm{HCO}_{2} \mathrm{NH}_{4}(129.5 \mathrm{mg}, 2.054 \mathrm{mmol})$ and $\mathrm{Pd} / \mathrm{C}(240 \mathrm{mg})$. The crude material was dissolved in $\mathrm{MeOH}$ and precipitated with $\mathrm{Et}_{2} \mathrm{O}$ to give $169.8 \mathrm{mg}$ (95\%) of $\mathbf{6 f}$. Colorless powder. ${ }^{1} \mathrm{H}-\mathrm{NMR}\left(\mathrm{D}_{2} \mathrm{O}\right):$ 7.54, $7.52(2 s, 2 \mathrm{NH}) ; 1.65,1.48,1.43,1.42(4 s$, 1:2:1:1, $\left.5 \mathrm{Me}_{2} \mathrm{C}\right) .{ }^{13} \mathrm{C}-\mathrm{NMR}\left(\mathrm{D}_{2} \mathrm{O}\right): 178.9,178.6,178.4,175.3$ (4s, $4 \mathrm{CO}$ (amide), $\mathrm{COOH}) ; 60.1,59.9,59.7,59.6(4 s, 5 \mathrm{C}(2)(\mathrm{Aib})) ; 27.2,27.1,26.9,26.5,25.9(5 q, 5$ $\left.M e_{2} \mathrm{C}\right)$. ESI-MS: $466\left(7,[M+\mathrm{Na}]^{+}\right), 444\left(100,[M+1]^{+}\right)$.

6.3. Cyclo(Aib-Aib-Aib-Aib-Aib) (7d). To a soln. of $6 \mathbf{6}(171.3 \mathrm{mg}, 0.386$ $\mathrm{mmol})$ in DMF (260 ml) were added DEPC (294.8 $\mathrm{mg}, 1.807 \mathrm{mmol})$ and DIEA (2.6 ml) at r.t. After stirring at r.t. for 2 weeks, DMF was removed in HV and subsequent $\mathrm{CC}\left(\mathrm{CH}_{2} \mathrm{Cl}_{2} / \mathrm{MeOH} 15: 1,3 \times\right)$ yielded $30.7 \mathrm{mg}$ of a mixture of $\mathbf{7 d}$ and an unknown compound. Additional CC (AcOEt/MeOH 20:1, 2×) gave 19.7 mg (12\%) 7d. Colorless powder. M.p. > 260. IR (KBr): 3420m, 3320s, 3120m, 2990m, 2980m, $2940 m, 1690 s, 1670 s, 1650 s, 1570 s, 1520 s, 1480 m, 1450 m, 1385 s, 1360 s, 1310 m$, $1280 s, 1220 s, 1210 s, 1185 m, 1170 m .{ }^{1} \mathrm{H}-\mathrm{NMR}\left(\mathrm{CDCl}_{3}\right): 6.63(s, 5 \mathrm{NH}) ; 1.54(s, 5$ $\left.\mathrm{Me}_{2} \mathrm{C}\right) .{ }^{13} \mathrm{C}-\mathrm{NMR}\left(\mathrm{CDCl}_{3}\right): 175.2(s, 5 \mathrm{CO}($ lactam $)) ; 58.0(s, 5 \mathrm{C}(2)(\mathrm{Aib})) ; 25.0(q, 5$ $\left.\mathrm{Me}_{2} \mathrm{C}\right)$. ESI-MS: $448\left(100,[\mathrm{M}+\mathrm{Na}]^{+}\right)$.

7. X-Ray Crystal-Structure Determination of $4 \mathbf{d}$ (see Table 3 and Fig. 2$)^{5}$ ). The measurements were made on a Rigaku AFC5R diffractometer using graphitemonochromated $\operatorname{Mo} K_{\alpha}$ radiation $(\lambda 0.71073 \AA$ ) and a $12 \mathrm{~kW}$ rotating anode

5) CCDC-1034190 contains the supplementary crystallographic data for this paper. These data can be obtained free of charge from The Cambridge Crystallographic Data Centre via www.ccdc.cam.ac.uk/data_request/cif. 
generator. The intensities were corrected for Lorentz and polarization effects. Azimuthal scans of several reflections indicated no need for an absorption correction. Equivalent reflections were merged. The data collection and refinement parameters are given in Table 3, and a view of the molecule is shown in Fig. 2. The structure was solved by direct methods using SHELXS86 [18], which revealed the positions of all non-H-atoms. The non-H-atoms were refined anisotropically. The amide $\mathrm{H}$-atoms were placed in the positions indicated by a difference electron density map and their positions were allowed to refine together with individual isotropic displacement parameters. All remaining $\mathrm{H}$-atoms were placed in geometrically calculated positions and refined by using a riding model where each $\mathrm{H}$-atom was assigned a fixed isotropic displacement parameter with a value equal to $1.2 \mathrm{U}_{\mathrm{eq}}$ of its parent $\mathrm{C}$-atom $\left(1.5 \mathrm{U}_{\mathrm{eq}}\right.$ for the Me groups). The refinement of the structure was carried out on $F^{2}$ by using full-matrix least-squares procedures, which minimized the function $\Sigma w\left(F_{\mathrm{o}}{ }^{2}-\right.$ $\left.F_{\mathrm{c}}{ }^{2}\right)^{2}$. A correction for secondary extinction was applied. Neutral atom scattering factors for non-H-atoms were taken from [19], and the scattering factors for $\mathrm{H}$-atoms were taken from [20]. Anomalous dispersion effects were included in $F_{\mathrm{c}}$ [21]; the values for $f$ and $f$ ' were those of [22]. The values of the mass attenuation coefficients are those of [23]. The SHELXL-2014 program [24] was used for all calculations.

\section{REFERENCES}

[1] F. S. Arnhold, A. Linden, H. Heimgartner, Helv. Chim. Acta 2014, 97, in press; doi: 10.1002/hlca.201400323.

[2] T. Degenkolb, W. Gams, H. Brückner, Chem. Biodivers. 2008, 5, 693. 
[3] a) J. Pastuszak, J. H. Gardner, J. Singh, D. H. Rich, J. Org. Chem. 1982, 47, 2982; b) T. Suga, S. Osada, H. Kodama, Pept. Sci. 2010, 47, 130; c) X.-H. Li, Y.-D. Wei, S.-M. Wang, M.-N. Wang, D.-W. Huang, Z.-L. Xiu, Chem. Res. Chinese Univ. 2012, 28, 1011.

[4] a) F. Rossi, M. Saviano, P. Di Talia, B. Di Blasio, C. Pedone, G. Zanotti, M. Mosca, G. Saviano, T. Tancredi, K. Ziegler, E. Benedetti, Biopolymers 1996, 40, 465; b) G. Zanotti, M. Saviano, G. Saviano, T. Tancredi, F. Rossi, C. Pedone, E. Benedetti, J. Pept. Res. 1998, 51, 460.

[5] a) E. Escudo, X. Vidal, X. Solans, E. Peggion, J. A. Subirana, J. Pept. Sci. 1996, 2, 59; b) J. Wang, S. Osada, H. Kodama, M. Kondo, Bull. Chem. Soc. Jpn. 2000, 73, 1221; c) Y. Demizu, S. Nagoya, M. Doi, Y. Sato, M. Tanaka, M. Kurihara, J. Org. Chem. 2012, 77, 9361.

[6] C. Cabrele, M. Langer, A. G. Beck-Sickinger, J. Org. Chem. 1999, 64, 4353.

[7] H. Heimgartner, Israel J. Chem. 1981, 21, 151; H. Heimgartner, Israel J. Chem. 1986, 27, 3; H. Heimgartner, in 'Amino Acids: Chemistry, Biology and Medicine, Eds. G. Lubec, G. A. Rosenthal, ESCOM, Leiden 1990, p. 29; H. Heimgartner, Angew. Chem., Int. Ed. Engl. 1991, 30, 238.

[8] a) D. Obrecht, H. Heimgartner, Helv. Chim. Acta 1981, 64, 482; b) P. Wipf, H. Heimgartner, Helv. Chim. Acta 1986, 69, 1153; c) D. Obrecht, H. Heimgartner, Helv. Chim. Acta 1987, 70, 102; d) P. Wipf, H. Heimgartner, Helv. Chim. Acta 1987, 70, 354; e) P. Wipf, H. Heimgartner, Helv. Chim. Acta 1988, 71, 140; f) P. Wipf, H. Heimgartner, Helv. Chim. Acta 1990, 73, 13.

[9] a) I. Dannecker-Dörig, A. Linden, H. Heimgartner, Collect. Czech. Chem. Commun. 2009, 74, 901; b) T. Jeremic, A. Linden, H. Heimgartner, Chem. Biodivers. 2004, 1, 1730; c) T. Jeremic, A. Linden, H. Heimgartner, Helv. 
Chim. Acta 2004, 87, 3056; d) T. Jeremic, A. Linden, K. Moehle, H. Heimgartner, Tetrahedron 2005, 61, 1871; e) T. Jeremic, A. Linden, H. Heimgartner, J. Pept. Sci. 2008, 14, 1051.

[10] a) B. V. V. Prasad, P. Balaram, Crit. Rev. Biochem. Mol. Biol. 1984, 16, 307; b) C. Toniolo, E. Benedetti, ISI Atlas Sci.: Biochem. 1988, 5, 1238; c) C. Toniolo, E. Benedetti, Macromolecules 1991, 24, 4004; d) C. Toniolo, Biopolymers 1989, 28, 247.

[11] a) I. L. Karle, P. Balaram, Biochemistry 1990, 29, 6747; b) E. Benedetti, M. Saviano, R. Iacovino, C. Pedone, A. Santini, M. Crisma, F. Formaggio, C. Toniolo, Q. B. Broxterman, J. Kamphuis, Biopolymers 1998, 46, 433.

[12] F. S. Arnhold, A. Linden, H. Heimgartner, Helv. Chim. Acta 2014, 97, 619.

[13] I. Dannecker-Dörig, A. Linden, H. Heimgartner, Helv. Chim. Acta 2011, 94, 993; I. Dannecker-Dörig, Ph. D. thesis, University of Zürich, 1995.

[14] C. K. Johnson, ORTEP II, Report ORNL-5138, Oak Ridge National Laboratory, Oak Ridge, Tennessee, 1976.

[15] J. Bernstein, R. E. Davis, L. Shimoni, N.-L. Chang, Angew. Chem. Int. Ed. 1995, 34, 1555.

[16] M. T. Leplawy, D. S. Jones, G. W. Kenner, R. C. Sheppard, Tetrahedron 1960, $11,39$.

[17] R. Nagaraj, P. Balaram, Heterocycles 1977, 7, 885.

[18] G. M. Sheldrick, Acta Crystallogr., Sect. A 1990, 46, 467.

[19] E. N. Maslen, A. G. Fox, M. A. O'Keefe, 'International Tables for Crystallography', Ed. A. J. C. Wilson, Kluwer Academic Publishers, Dordrecht, 1992, Vol. C, Table 6.1.1.1, p. 477.

[20] R. F. Stewart, E. R. Davidson, W. T. Simpson, J. Chem. Phys. 1965, 42, 3175. 
[21] J. A. Ibers, W. C. Hamilton, Acta Crystallogr. 1964, 17, 781.

[22] D. C. Creagh, W. J. McAuley, 'International Tables for Crystallography', Ed. A. J. C. Wilson, Kluwer Academic Publishers, Dordrecht, 1992, Vol. C, Table 4.2.6.8, p. 219 .

[23] D. C. Creagh, J. H. Hubbell, 'International Tables for Crystallography', Ed. A. J. C. Wilson, Kluwer Academic Publishers, Dordrecht, 1992, Vol. C, Table 4.2.4.3, p. 200.

[24] G. M. Sheldrick, Acta Crystallogr., Sect. A 2008, 64, 112. 


\section{Legends}

Fig. 1. Structures of the cyclopentapeptides $\mathbf{7 a}$ and $\mathbf{7 b}$.

Fig. 2. ORTEP Plot [14] of the molecular structure of the pentapeptide $4 \mathbf{d}$ (50\% probability ellipsoids, arbitrary numbering of atoms, $\mathrm{H}$-atoms bonded to $\mathrm{C}$-atoms omitted for clarity)

Table 1. Intra- and intermolecular H-bonds of pentapeptide $4 \mathbf{d}$ (atom numbering refers to Fig. 2)

Table 2. Torsion angles $\phi, \psi$, and $\omega\left[^{\circ}\right]$ of the backbone of $\mathbf{4 d}$ in the crystal

Table 3. Crystallographic Data for Compound $\mathbf{4 d}$ 
Table 1. Intra- and intermolecular H-bonds of pentapeptide $\mathbf{4 d}$ (atom numbering refers to Fig. 2)

\begin{tabular}{|llll|}
\hline Donor ${ }^{\cdots}$ Acceptor & $\mathrm{N}^{\cdots} \mathrm{O}[\AA]$ & $\mathrm{H}^{\cdots} \mathrm{O}[\AA]$ & $\mathrm{N}-\mathrm{H}^{\cdots} \mathrm{O}\left[^{\circ}\right]$ \\
\hline $\mathrm{N}(1)-\mathrm{H}^{\cdots} \mathrm{O}(8)$ & $2.952(3)$ & $2.10(3)$ & $167(2)$ \\
$\mathrm{N}(4)-\mathrm{H}^{\cdots} \mathrm{O}(11)$ & $3.053(3)$ & $2.21(3)$ & $169(2)$ \\
$\mathrm{N}(7)-\mathrm{H}^{\cdots} \mathrm{O}(14)$ & $2.879(3)$ & $2.05(3)$ & $158(2)$ \\
$\mathrm{N}(10)-\mathrm{H}^{\cdots} \mathrm{O}\left(24^{\prime}\right)$ & $3.053(3)$ & $2.21(3)$ & $164(2)$ \\
$\mathrm{N}(13)-\mathrm{H}^{\cdots} \mathrm{O}\left(2^{\prime}\right)$ & $2.860(3)$ & $2.03(3)$ & $168(2)$ \\
& & & \\
\hline
\end{tabular}

Table 2. Torsion angles $\phi, \psi$, and $\omega\left[^{\circ}\right]$ of the backbone of $\mathbf{4} \mathbf{d}$ in the crystal<smiles>C#CC(=O)NC(C)(C)C(=O)NC(C)(C)C(=O)NC(=O)OCc1ccccc1</smiles>

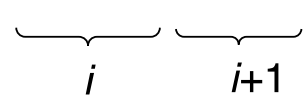

\begin{tabular}{|llllllll|}
\hline$\phi_{(\mathrm{i})}$ & $\psi_{(\mathrm{i})}$ & $\omega_{(\mathrm{i})}$ & $\phi_{(\mathrm{i}+1)}$ & $\psi_{(\mathrm{i}+1)}$ & $\omega_{(\mathrm{i}+1)}$ & & \\
\hline$-54.1(3)$ & $-42.1(3)$ & $-177.3(2)$ & $-55.2(3)$ & $-27.5(3)$ & $-179.3(2)$ & & \\
\hline$\phi_{(\mathrm{i}+2)}$ & $\psi_{(\mathrm{i}+2)}$ & $\omega_{(\mathrm{i}+2)}$ & $\phi_{(\mathrm{i}+3)}$ & $\psi_{(\mathrm{i}+3)}$ & $\omega_{(\mathrm{i}+3)}$ & $\phi_{(\mathrm{i}+4)}$ & $\psi_{(\mathrm{i}+4)}$ \\
\hline$-58.4(3)$ & $-23.7(3)$ & $174.9(2)$ & $-60.4(3)$ & $-20.2(3)$ & $-176.9(2)$ & $55.1(3)$ & $44.5(3)$ \\
\hline
\end{tabular}


Table 3. Crystallographic Data for Compound 4d

\begin{tabular}{|c|c|c|c|}
\hline \multicolumn{3}{|c|}{ Crystallized from } & AcOEt/hexane \\
\hline \multicolumn{3}{|c|}{ Empirical formula } & $\mathrm{C}_{27} \mathrm{H}_{41} \mathrm{~N}_{5} \mathrm{O}_{8}$ \\
\hline \multicolumn{3}{|c|}{ Formula weight } & 563.65 \\
\hline \multicolumn{3}{|c|}{ Crystal color, habit } & colorless, needle \\
\hline \multicolumn{3}{|c|}{ Crystal dimensions [mm] } & $0.20 \times 0.25 \times 0.45$ \\
\hline \multicolumn{3}{|c|}{ Temperature $[\mathrm{K}]$} & 173(1) \\
\hline \multicolumn{3}{|c|}{ Crystal system } & monoclinic \\
\hline \multicolumn{3}{|c|}{ Space group } & $P 2_{1} / n$ \\
\hline \multicolumn{3}{|l|}{$Z$} & 4 \\
\hline \multicolumn{3}{|c|}{ Reflections for cell determination } & 25 \\
\hline \multicolumn{3}{|c|}{$2 \theta$ range for cell determination $\left[{ }^{\circ}\right]$} & $20-37$ \\
\hline \multirow{5}{*}{\multicolumn{2}{|c|}{ Unit cell parameters }} & $a[\AA]$ & $11.674(2)$ \\
\hline & & $b[\AA]$ & $16.174(2)$ \\
\hline & & $c[\AA]$ & $16.774(2)$ \\
\hline & & $\beta\left[^{\circ}\right]$ & $103.29(1)$ \\
\hline & & $V\left[\AA^{3}\right]$ & $3082.1(8)$ \\
\hline \multicolumn{3}{|c|}{$D_{x}\left[\mathrm{~g} \mathrm{~cm}^{-3}\right]$} & 1.215 \\
\hline \multicolumn{3}{|c|}{$\mu(\mathrm{MoK} \alpha)\left[\mathrm{mm}^{-1}\right]$} & 0.090 \\
\hline \multicolumn{3}{|c|}{ Scan type } & $\omega / 2 \theta$ \\
\hline \multicolumn{3}{|c|}{$2 \theta_{(\max )}\left[^{\circ}\right]$} & 55 \\
\hline \multicolumn{3}{|c|}{ Total reflections measured } & 7404 \\
\hline \multicolumn{3}{|c|}{ Symmetry independent reflections } & 7068 \\
\hline \multicolumn{3}{|c|}{ Reflections with $I>2 \sigma(I)$} & 3881 \\
\hline \multicolumn{3}{|c|}{ Reflections used in refinement } & 7068 \\
\hline \multicolumn{3}{|c|}{ Parameters refined } & 391 \\
\hline \multirow[t]{2}{*}{ Final } & $R(F)[I>2 \sigma$ & reflections] & 0.0530 \\
\hline & $w R\left(F^{2}\right)($ all d & & 0.1412 \\
\hline \multicolumn{3}{|c|}{ Weighting parameter $\left.(a)^{\mathrm{a}}\right)$} & 0.0513 \\
\hline \multicolumn{3}{|c|}{ Goodness of fit } & 1.003 \\
\hline \multicolumn{3}{|c|}{ Secondary extinction coefficient } & $0.0018(5)$ \\
\hline \multicolumn{3}{|c|}{ Final $\Delta_{\max } / \sigma$} & 0.000 \\
\hline \multicolumn{3}{|c|}{$\Delta \rho(\max ; \min )\left[\mathrm{e} \AA^{-3}\right]$} & $0.25 ;-0.23$ \\
\hline
\end{tabular}

a) $\quad w^{-1}=\sigma^{2}\left(F_{\mathrm{o}}{ }^{2}\right)+(a P)^{2}$, where $P=\left(F_{\mathrm{o}}{ }^{2}+2 F_{\mathrm{c}}{ }^{2}\right) / 3$ 
Scheme 1

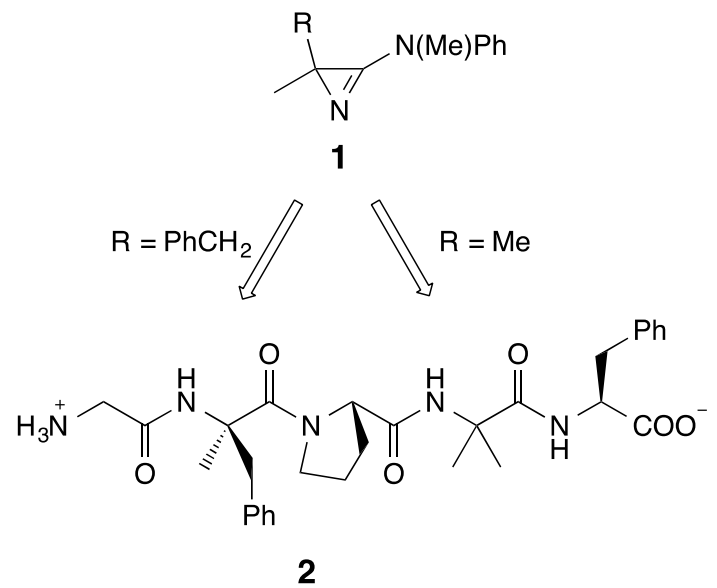

2

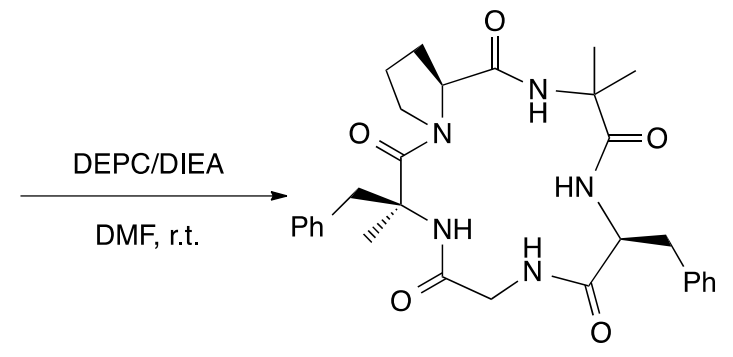

3

Scheme 2

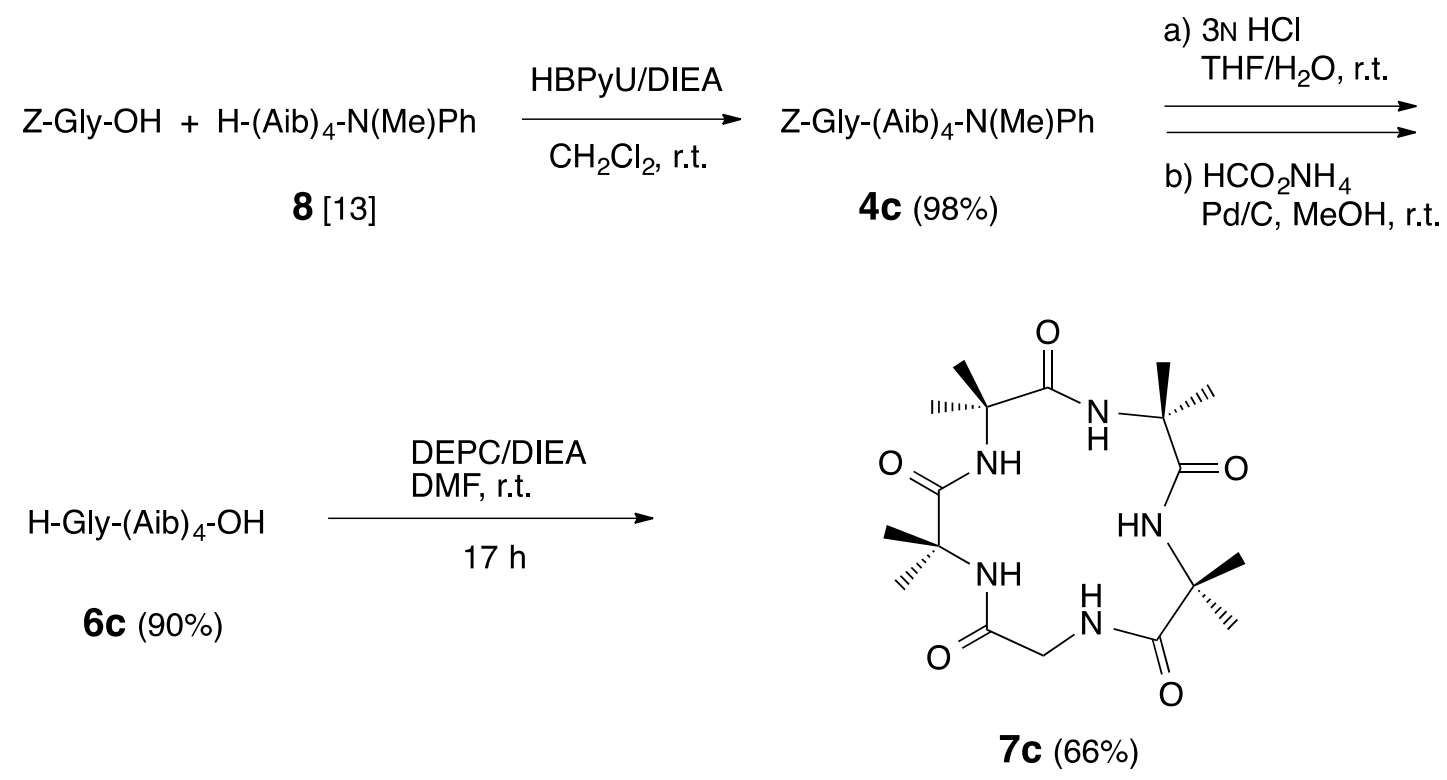


Scheme 3

$$
\begin{aligned}
& \text { a) HBPyU, DIEA } \\
& \mathrm{CH}_{2} \mathrm{Cl}_{2} \text {, r.t. } \\
& \text { Z-(Aib) }{ }_{2}-\mathrm{OH}+\mathrm{H} \text {-Gly-OMe } \\
& 9 \text { [13] } \\
& \text { b) } \mathrm{LiOH} \cdot \mathrm{H}_{2} \mathrm{O} \\
& \mathrm{MeOH} \\
& \text { Z-(Aib) })_{2}-\mathrm{Gly}-\mathrm{OH} \\
& 10(86 \%)
\end{aligned}
$$

HBPyU, DIEA

$$
\begin{aligned}
& 10+\mathrm{H}-(\text { Aib })_{2}-\mathrm{X} \stackrel{\mathrm{CH}_{2} \mathrm{Cl}_{2} \text {, r.t. }}{\longrightarrow} \mathrm{Z} \text {-(Aib) }{ }_{2}-\mathrm{Gly}-(\mathrm{Aib})_{2}-\mathrm{X} \\
& \begin{aligned}
\text { 11a } X & =\mathrm{OMe} & \text { 4d } X & =\mathrm{OMe}(74 \%) \\
\text { b } X & =\mathrm{N}(\mathrm{Me}) \mathrm{Ph} & \text { e } X & =\mathrm{N}(\mathrm{Me}) \mathrm{Ph}(81 \%)
\end{aligned}
\end{aligned}
$$

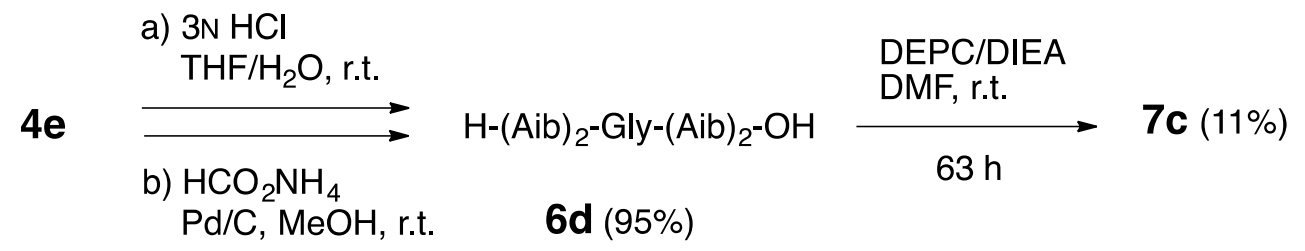

Scheme 4
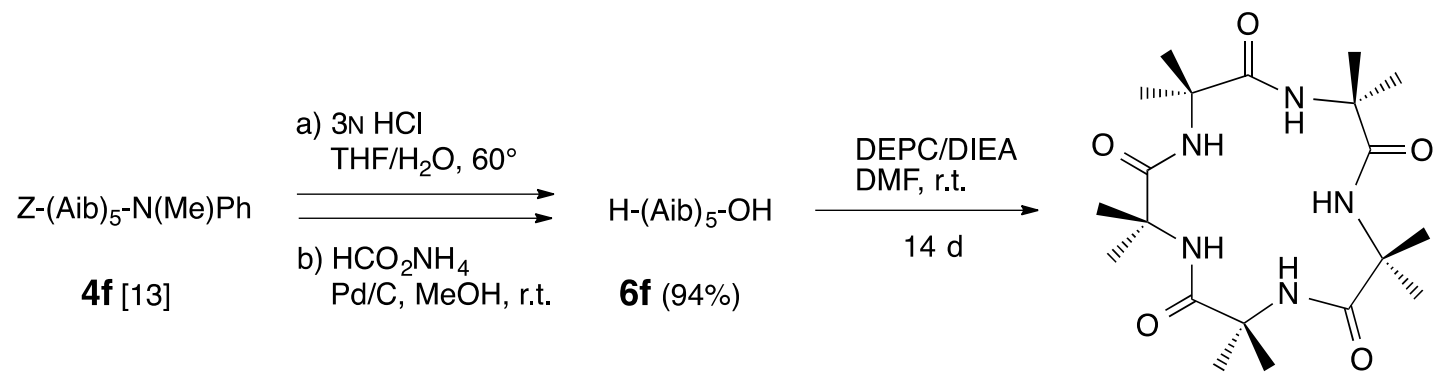

7d (12\%) 
Figure 1

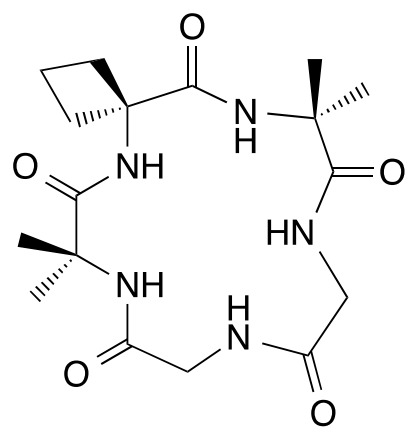

$7 a$

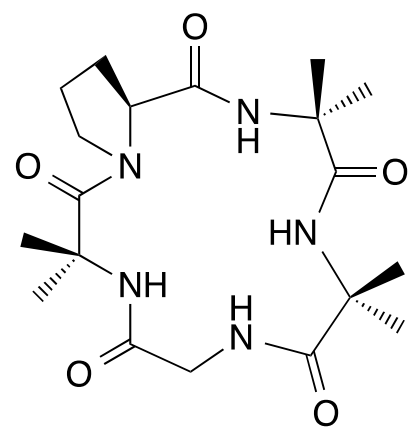

7b

Figure 2

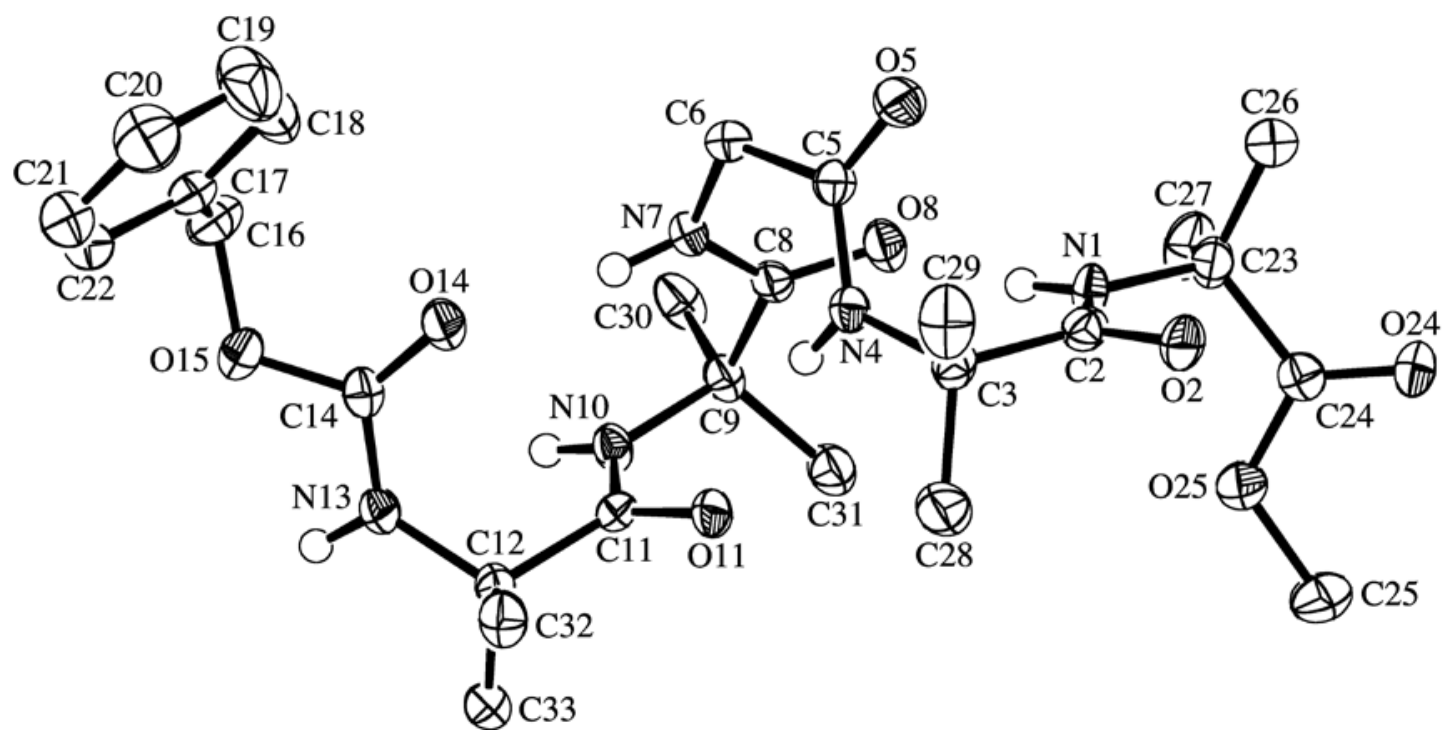


Graphical Abstract

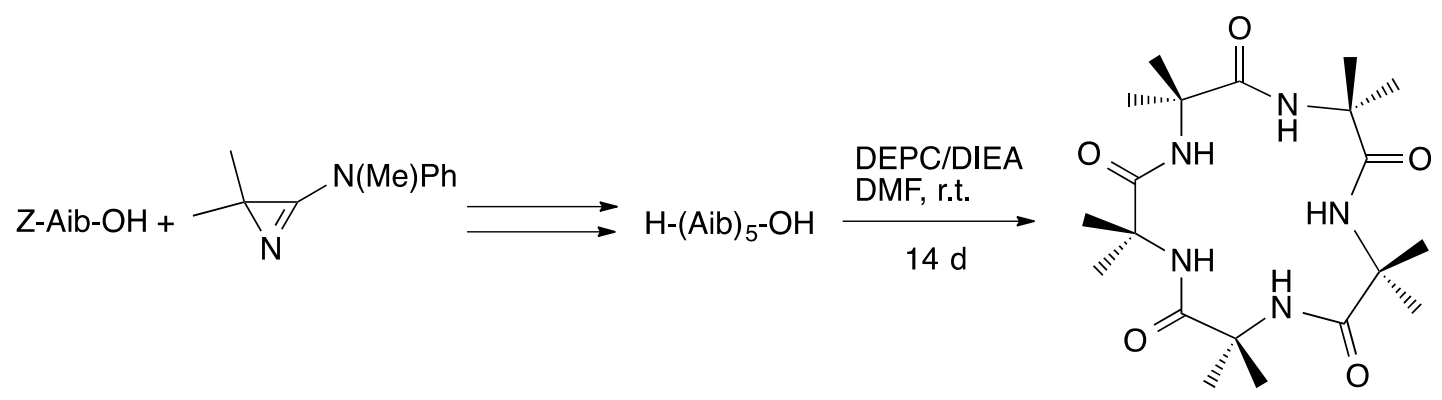

